\title{
Non-supersymmetric membrane flows from fake supergravity and multi-trace deformations
}

\author{
loannis Papadimitriou \\ DESY Theory Group, \\ Notkestrasse 85, D-22603 Hamburg, Germany, and \\ Center for Mathematical Physics, \\ Bundesstrasse 55, D-20146 Hamburg, Germany \\ E-mail: ioannis.papadimitriou@desy.de
}

\begin{abstract}
We use fake supergravity as a solution generating technique to obtain a continuum of non-supersymmetric asymptotically $A d S_{4} \times S^{7}$ domain wall solutions of elevendimensional supergravity with non-trivial scalars in the $\mathrm{SL}(8, \mathbb{R}) / \mathrm{SO}(8)$ coset. These solutions are continuously connected to the supersymmetric domain walls describing a uniform sector of the Coulomb branch of the M2-brane theory. We also provide a general argument that under certain conditions identifies the fake superpotential with the exact large- $N$ quantum effective potential of the dual theory, describing a marginal multi-trace deformation. This identification strongly motivates further study of fake supergravity as a solution generating method and it allows us to interpret our non-supersymmetric solutions as a family of marginal triple-trace deformations of the Coulomb branch that completely break supersymmetry and to calculate the exact large- $N$ anomalous dimensions of the operators involved. The holographic one- and two-point functions for these solutions are also computed.
\end{abstract}

KeYwords: Gauge-gravity correspondence, AdS-CFT Correspondence, Field Theories in Lower Dimensions, M-Theory. 


\section{Contents}

1. Introduction and summary of results

2. The $\mathrm{SL}(N, \mathbb{R}) / \mathrm{SO}(N)$ sector of gauged maximal supergravity and its higher-dimensional origin

3. All asymptotically AdS Poincaré domain walls of the $\operatorname{SL}(N, \mathbb{R}) / \operatorname{SO}(N)$ sector

4. The non-supersymmetric Poincaré domain walls in $D=4$

4.1 Domain walls with a single scalar

4.2 Exact closed form solutions

5. Exact non-supersymmetric membrane flows

6. Holographic one-point functions

$6.1 \Delta=\Delta_{+}$

$6.2 \Delta=\Delta_{-}$

7. Holographic two-point functions

8. The fake superpotential as a quantum effective potential and multi-trace deformations

8.1 Triple-trace deformation of the Coulomb branch

A. Explicit form of the domain wall metric for $W(\phi ; \alpha)$, to first order in $\alpha-\alpha_{o}$ and for general $k$

B. Uplifting the MTZ black hole to eleven dimensions

G. Computation of the holographic two-point functions

D. Multi-trace deformations in the large- $N$ limit and the AdS/CFT correspondence

\section{Introduction and summary of results}

The study of domain wall solutions of various supergravity theories has been strongly motivated in recent years by the role these play in a variety of physical contexts, from the AdS/CFT correspondence, where they describe an RG flow of the conformal field theory 
residing on the conformal boundary of AdS, to 'Brane World' scenarios and cosmological models (see [1] for an extensive review of domain walls of $N=1$ supergravity in four dimensions). Although, when they arise as solutions to a particular supergravity theory, such domain walls are often supersymmetric, this need not be the case. Indeed, many non-supersymmetric gravitational theories admit domain wall solutions as well. In this paper, however, we will emphasize the fact that true supergravity theories also admit nonsupersymmetric domain wall solutions, which can be physically important.

We will focus on domain walls preserving Poincaré invariance in $d=D-1$ dimensions, where $D$ is the spacetime dimension where the given gravitational theory lives. Such domain walls take the form ${ }^{1}$

$$
d s_{D}^{2}=d r^{2}+e^{2 A(r)} \eta_{i j} d x^{i} d x^{j}, \quad \phi^{I}=\phi^{I}(r),
$$

where $\eta=\operatorname{diag}(-1,1, \ldots, 1)$ is the Minkowski metric in $d$ dimensions. Since only the metric and a number of scalar fields are involved in these solutions, they can generically be described by an effective gravitational theory with an action of the form

$$
S=\int_{\mathcal{M}} d^{D} x \sqrt{-g}\left(\frac{1}{2 \kappa_{D}^{2}} R-\frac{1}{2} \mathcal{G}_{I J}(\phi) g^{\mu \nu} \partial_{\mu} \phi^{I} \partial_{\nu} \phi^{J}-V(\phi)\right),
$$

where $\kappa_{D}^{2}=8 \pi G_{D}$ is the effective gravitational constant and $\mathcal{G}_{I J}$ is a generic (Riemannian) metric on the scalar manifold. Such theories arise naturally as consistent truncations of various gauged supergravities, in which case the scalar potential is generated by the nontrivial gauging of (some of) the isometries of the scalar manifold. Generically, however, this effective description will only be valid locally in the moduli space of a given supergravity theory [3]. Here we are interested in the application of domain walls to the AdS/CFT correspondence and so we assume that the metric (1.1) is asymptotically AdS, which is equivalent to the statement that $A(r) \sim r$ as $r \rightarrow \infty$. This in turn implies that the scalar potential $V(\phi)$ has at least one stable fixed point at $\phi^{I}=\phi_{*}^{I}$ such that $V\left(\phi_{*}\right)<0$. By a reparameterization of the scalar manifold we can set $\phi_{*}^{I}=0$. If this potential arises from some gauged supergravity, this fixed point corresponds to the maximally symmetric $A d S_{D}$ vacuum.

The equations of motion following from the action (1.2) are Einstein's equations

$$
R_{\mu \nu}-\frac{1}{2} R g_{\mu \nu}=\kappa_{D}^{2} T_{\mu \nu}
$$

with the stress tensor given by

$$
T_{\mu \nu}=\mathcal{G}_{I J}(\phi) \partial_{\mu} \phi^{I} \partial_{\nu} \phi^{J}-g_{\mu \nu}\left(\frac{1}{2} \mathcal{G}_{I J}(\phi) g^{\rho \sigma} \partial_{\rho} \phi^{I} \partial_{\sigma} \phi^{J}+V(\phi)\right)
$$

and

$$
\nabla^{\mu}\left(\mathcal{G}_{I J}(\phi) \partial_{\mu} \phi^{J}\right)-\frac{1}{2} \frac{\partial \mathcal{G}_{L M}}{\partial \phi^{I}} g^{\mu \nu} \partial_{\mu} \phi^{L} \partial_{\nu} \phi^{M}-\frac{\partial V}{\partial \phi^{I}}=0
$$

\footnotetext{
${ }^{1}$ More general domain walls with a different isometry do exist, as is discussed e.g. in [月], but we will not discuss them here.
} 
Substituting the domain wall ansatz (1.1) into the equations of motion one obtains the following equations for the warp factor $A(r)$ and the scalar fields $\phi^{I}(r)$ :

$$
\begin{aligned}
\dot{A}^{2}-\frac{\kappa^{2}}{d(d-1)}\left(\mathcal{G}_{I J}(\phi) \dot{\phi}^{I} \dot{\phi}^{J}-2 V(\phi)\right) & =0, \\
\ddot{A}+d \dot{A}^{2}+\frac{2 \kappa^{2}}{d-1} V(\phi) & =0, \\
\mathcal{G}_{I J}(\phi) \ddot{\phi}^{J}+\frac{\partial \mathcal{G}_{I J}}{\partial \phi^{K}} \dot{\phi}^{K} \dot{\phi}^{J}-\frac{1}{2} \frac{\partial \mathcal{G}_{L M}}{\partial \phi^{I}} \dot{\phi}^{L} \dot{\phi}^{M}+d \dot{A} \mathcal{G}_{I J}(\phi) \dot{\phi}^{J}-\frac{\partial V}{\partial \phi^{I}} & =0,
\end{aligned}
$$

where the dot denotes the derivative with respect to the radial coordinate $r$.

It is important to distinguish between two types of solutions of these second order equations. Following [ [- we will call a 'BPS domain wall' any domain wall of the form (1.1) which satisfies the first order equations

$$
\begin{aligned}
\dot{A} & =-\frac{\kappa^{2}}{d-1} W(\phi), \\
\dot{\phi}^{I} & =\mathcal{G}^{I J}(\phi) \frac{\partial W}{\partial \phi^{J}},
\end{aligned}
$$

for some function $W(\phi)$ of the scalar fields such that the scalar potential can be expressed as

$$
V(\phi)=\frac{1}{2}\left(\mathcal{G}^{I J}(\phi) \frac{\partial W}{\partial \phi^{I}} \frac{\partial W}{\partial \phi^{J}}-\frac{d \kappa^{2}}{d-1} W^{2}\right) .
$$

Note that the first order equations (11.7) together with (1.8) ensure that the second order equations (1.6) are automatically satisfied. Given the expression (1.8) for the scalar potential in terms of the function $W$, the first order equations (1.7) can be derived á la Bogomol'nyi by extremizing the energy functional $E[A, \phi]$ that has $(1.6)$ as its Euler-Lagrange

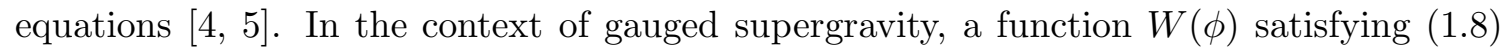
arises naturally as the superpotential, $W_{o}(\phi)$, which enters the gravitino and dilatino variations

$$
\begin{aligned}
& \delta \psi_{\mu}=D_{\mu} \varepsilon-\frac{\kappa^{2}}{2(d-1)} W_{o}(\phi) \gamma_{\mu} \varepsilon \\
& \delta \chi^{I}=\left(\gamma^{\mu} \partial_{\mu} \phi^{I}+\mathcal{G}^{I J}(\phi) \frac{\partial W_{o}}{\partial \phi^{J}}\right) \varepsilon .
\end{aligned}
$$

It follows that the domain walls defined by the superpotential $W_{o}(\phi)$ are supersymmetric solutions of the particular gauged supergravity. Crucially, however, equation (1.8) does not define the function $W(\phi)$ uniquely and hence there may generically exist other functions $W(\phi)$ satisfying (1.8) in addition to $W_{o}(\phi) .{ }^{2}$ This has been termed fake supergravity and in this context any function $W(\phi)$ that solves (1.8) is called a fake superpotential [2, 3, 6, 7]. In [7] it was shown that any BPS Poincaré domain wall of the form (1.1), defined by a function $W(\phi)$ which is not necessarily the true superpotential of a given gauged supergravity,

\footnotetext{
${ }^{2}$ Note, however, that not every function $W(\phi)$ that satisfies $(1.8)$ is acceptable, since it will not generically correspond to an asymptotically AdS domain wall. We will discuss in detail the conditions $W(\phi)$ must satisfy below. See also 2, 勾.
} 
is 'supersymmetric' in the sense that one can always find Killing spinors, at least locally. In [7] this was considered as an indication that any function $W(\phi)$ that solves (1.8) (and possibly subject to suitable boundary conditions) may be the true superpotential of some supergravity theory, even though there is no systematic way to find which is the relevant, known or unknown, theory [8]. Despite the elegance of this statement, it is difficult in practice to confirm or refute it. We will adopt a rather different point of view here, however. Namely, we will confine ourselves to a particular gauged supergravity, with a certain superpotential $W_{o}(\phi)$. Clearly, any BPS domain wall defined by a solution $W(\phi) \neq W_{o}(\phi)$ of (1.8) is not supersymmetric in this context. We will nevertheless continue to call such solutions 'BPS' since they satisfy the first order equations (1.7). They are still special solutions because they allow for the definition of fake Killing spinors via (1.9) with $W_{o}(\phi)$ replaced by the fake superpotential $W(\phi)[2]{ }^{3}$ The existence of fake Killing spinors implies, in particular, non-perturbative gravitational stability, at least in the absence of naked singularities [9, 4, 2].

If the scalar potential cannot be written in the form (1.8), however, there can still exist domain wall solutions of the form (1.1) that solve the second order equations (1.6). We will refer to such solutions as 'non-BPS domain walls'. ${ }^{4}$ We will not consider further such domain walls here since we are interested in scalar potentials that arise from gauged supergravities, and such potentials are guaranteed to be expressible in the form (1.8) since this is at least possible using the true superpotential $W_{o}(\phi) .^{5}$

Although one often views fake supergravity as an effective subsector of some gauged supergravity, by identifying both the scalar potential and the fake superpotential of fake supergravity with the true potential and superpotential respectively of the gauged supergravity [4, 3, 6], we will instead treat fake supergravity as a powerful solution generating technique for non-supersymmetric solutions of a given gauged supergravity. In particular, we will treat (1.8) as a first order non-linear differential equation for the fake superpotential $W(\phi)$ [10, 11, 2, 12] (see also [13] where a very similar perspective is adopted). For scalar potentials arising from some gauged supergravity this equation admits at least one solution, namely the true superpotential of the theory. Our aim here will be to determine all solutions of (1.8) satisfying appropriate boundary conditions. Each solution $W(\phi) \neq W_{o}(\phi)$ defines a non-supersymmetric domain wall solution of the given gauged supergravity, and therefore describes a non-supersymmetric RG flow of the dual field theory.

The paper is organized as follows. In the next section we will discuss a common subsector of gauged maximal supergravities in dimensions $D=4,5,7$ with the scalar fields parameterizing an $\mathrm{SL}(N, \mathbb{R}) / \mathrm{SO}(N)$ coset, where $N=8,6,5$ respectively. The complete non-linear ansatz for uplifting any solution of this subsector to eleven-dimensional or Type IIB supergravity is known [14, 15], and all supersymmetric Poincaré domain walls, describ-

\footnotetext{
${ }^{3}$ Note that our fake superpotential differs by a factor of $-\frac{2(d-1)}{\kappa^{2}}$ relative to the fake superpotential defined in 22. The supercovariant derivative is uniquely determined, however, by the requirement that it reduces to that of pure AdS, namely $\left(D_{\mu}+\frac{1}{2 l} \gamma_{\mu}\right) \varepsilon$, when the scalar fields vanish.

${ }^{4}$ Note though the analysis of [1], which suggests that any potential that admits domain wall solutions can be written in the form (1.8) and so there are no 'non-BPS' domain walls.

${ }^{5}$ Generically the superpotential $W_{o}(\phi)$ will be a matrix, however, instead of a scalar quantity. See e.g. 2].
} 
ing a uniform subsector of the Coulomb branch of respectively the $M 2-, D 3-$, or $M 5$-brane theory have been constructed [16-18, 14, 5]. In section 3 we solve equation (1.8) with the scalar potential of gauged supergravity as a differential equation for the fake superpotential $W(\phi)$, subject to suitable boundary conditions. We show that analytic non-supersymmetric solutions exist only in dimension $D=4$, while the superpotential $W_{o}$ is the only analytic solution of (1.8) for $D=5,7$. In section 1 we systematically discuss how to obtain these non-supersymmetric solutions in four dimensions in closed form by consistently reducing the number of scalar fields, and we solve (1.8) exactly, obtaining a family of exact fake superpotentials, for a special case involving a single scalar field. We then uplift this solution to eleven dimensions in section 5 using the ansatz discussed in section 2 and, noting that the MTZ black hole [19] in four dimensions is interestingly a solution of exactly the same action as our exact domain wall, we also give the eleven-dimensional black hole solution (given explicitly in appendix B). The holographic one- and two-point functions for the non-supersymmetric domain walls are then computed respectively in sections 6 and 7 . Finally, in section 8 we show that under certain circumstances the fake superpotential $W(\phi)$ that solves (1.8) and corresponds to an asymptotically AdS domain wall, defines a marginal multi-trace deformation of the dual field theory. This means that solving equation (1.8) as a differential equation for the fake superpotential not only is interesting as a method for finding exact non-supersymmetric supergravity solutions, but also, these solutions can often be interpreted as the exact holographic duals of a marginal multi-trace deformation of the boundary theory. Applying this observation to the non-supersymmetric domain walls we have constructed leads to the conclusion that they correspond to a continuous family of marginal triple-trace deformations of the Coulomb branch of the M2-brane theory. A number of technical results are collected in the appendices.

\section{The $\operatorname{SL}(N, \mathbb{R}) / \mathrm{SO}(N)$ sector of gauged maximal supergravity and its higher-dimensional origin}

The scalar manifold of $D$-dimensional maximal supergravity is the coset $E_{11-D(11-D)} / K$, where $E_{n(n)}$ is the maximally non-compact form of the exceptional Lie group $E_{n}$ and $K$ is its maximal compact subgroup. ${ }^{6}$ Following 18,14 , 5, we specialize to an $\operatorname{SL}(N, \mathbb{R})$ subgroup of $E_{11-D}$, where $N=4(D-2) /(D-3)$, and consider the $\frac{1}{2} N(N+1)-1$ scalars of the coset $\mathrm{SL}(N, \mathbb{R}) / \mathrm{SO}(N)$. This scalar sector is common to all maximal supergravities in any dimension. In particular, for $D=7$ we consider $\operatorname{SL}(5, \mathbb{R}) \cong E_{4}$, for $D=5 \mathrm{SL}(6, \mathbb{R}) \subset E_{6}$ and for $D=4 \mathrm{SL}(8, \mathbb{R}) \subset E_{7}$.

The Lagrangian density describing this sector of the gauged version of maximal supergravity in $D$ dimensions, where the $\mathrm{SO}(N)$ symmetry is gauged, is

$$
e^{-1} \mathcal{L}_{D}=\frac{1}{2 \kappa_{D}^{2}} R+\frac{1}{8 \kappa_{D}^{2}} \operatorname{tr}\left(\partial_{\mu} \mathcal{M} \partial^{\mu} \mathcal{M}^{-1}\right)-V
$$

\footnotetext{
${ }^{6}$ For $n<6$ the following identifications are made $E_{5} \cong D_{5}, E_{4} \cong A_{4}, E_{3} \cong A_{2} \times A_{1}, E_{2} \cong A_{1} \times \mathbb{R}$ and $E_{1} \cong \mathbb{R}$.
} 
where $\mathcal{M}=S^{T} S$ is a symmetric $N \times N$ matrix, with $S$ in the fundamental representation of $\operatorname{SL}(N, \mathbb{R})$, and the potential $V$ takes the form ${ }^{7}$

$$
V=-\frac{(D-3)^{2}}{16 \kappa_{D}^{2} l_{D}^{2}}\left[(\operatorname{tr} \mathcal{M})^{2}-2 \operatorname{tr}\left(\mathcal{M}^{2}\right)\right]
$$

In these expressions the trace is taken in the fundamental of $\mathrm{SL}(N, \mathbb{R})$. Using an $\mathrm{SO}(N)$ rotation, the matrix $\mathcal{M}$ can be diagonalized so that

$$
\mathcal{M}=\operatorname{diag}\left(X_{1}, \ldots, X_{N}\right)
$$

where the $N$ scalars $X_{i}$ satisfy the constraint

$$
\operatorname{det} \mathcal{M}=\prod_{i=1}^{N} X_{i}=1
$$

It might be useful to note that in terms of the non-trivial scalars that we have kept at this point, the symmetric tensor $T_{i j}$ parameterizing the full scalar manifold of the maximal supergravity takes the form $T_{i j}=X_{i} \delta_{i j}$. The $N$ constrained scalars $X_{i}$ can be parameterized by $N-1$ independent scalar fields, $\varphi^{I}, I=1, \ldots, N-1$, as

$$
X_{i}=e^{-\frac{1}{2} \vec{b}_{i} \cdot \vec{\varphi}}
$$

where the $N$ vectors $\vec{b}_{i}$ are (up to a factor of 2) the weight vectors of the fundamental representation of $\mathrm{SL}(N, \mathbb{R})$ and they satisfy

$$
\vec{b}_{i} \cdot \vec{b}_{j}=8 \delta_{i j}-\frac{8}{N}, \quad \sum_{i=1}^{N} \vec{b}_{i}=0, \quad \sum_{i=1}^{N} b_{i I} b_{i J}=8 \delta_{I J} .
$$

After diagonalizing the matrix $\mathcal{M}$ and dropping the kinetic terms for the original off-diagonal scalars which decouple, the Lagrangian (2.1) becomes

$$
e^{-1} \mathcal{L}_{D}=\frac{1}{2 \kappa_{D}^{2}} R-\frac{1}{4 \kappa_{D}^{2}} \sum_{I=1}^{N-1} \partial_{\mu} \varphi^{I} \partial^{\mu} \varphi^{I}-V,
$$

where the potential is now given by

$$
V=-\frac{(D-3)^{2}}{16 \kappa_{D}^{2} l_{D}^{2}}\left(\left(\sum_{i=1}^{N} X_{i}\right)^{2}-2 \sum_{i=1}^{N} X_{i}^{2}\right) .
$$

This Lagrangian, which is a special case of (1.2), falls into the framework of fake supergravity described in the previous section. In order to make contact with our notation in the previous section we also define the rescaled scalars

$$
\phi^{I} \equiv \frac{1}{\sqrt{2} \kappa_{D}} \varphi^{I},
$$

\footnotetext{
${ }^{7}$ Note that the $A d S_{D}$ radius, $l_{D}$, is related to the coupling $g$ in 14 , 15] by $l_{D}=(D-3) / 2 g$.
} 
which have a canonically normalized kinetic term.

The equations of motion for this gravity-scalar system can be written as

$$
\begin{aligned}
R_{\mu \nu} & =\frac{1}{4} \sum_{i=1}^{N} X_{i}^{-2} \partial_{\mu} X_{i} \partial_{\nu} X_{i}+\frac{2 \kappa_{D}^{2}}{D-2} V g_{\mu \nu}, \\
\square \log X_{i} & =\frac{(D-3)^{2}}{2 l_{D}^{2}}\left(2 X_{i}^{2}-X_{i} \sum_{j=1}^{N} X_{j}-\frac{2}{N} \sum_{j=1}^{N} X_{j}^{2}+\frac{1}{N}\left(\sum_{j=1}^{N} X_{j}\right)^{2}\right) .
\end{aligned}
$$

The second of these equations can be derived by starting from the equation of motion for the independent scalar fields $\varphi^{I}$,

$$
\square \varphi^{I}=\frac{(D-3)^{2}}{8 l_{D}^{2}} \sum_{i=1}^{N} b_{i I} X_{i}\left(\sum_{j=1}^{N} X_{j}-2 X_{i}\right),
$$

noticing that the last equation in (2.6) implies that

$$
\square \varphi^{I}=-\frac{1}{4} \sum_{i=1}^{N} b_{i I} \log X_{i},
$$

and adding a term to ensure that the sum over $i$ is zero, in agreement with the constraint (2.4).

The gravity-scalar theory we have just discussed was obtained as a consistent truncation of gauged maximal supergravity in $D$ dimensions. However, the maximal gauged supergravities in $D=4$ and $D=7$ are known to arise themselves as consistent truncations to the massless fields of the Kaluza-Klein compactification of eleven-dimensional supergravity on $S^{7}$ and $S^{4}$ respectively [20-22]. Moreover, the gauged maximal supergravity in $D=5$ is also believed to arise as an $S^{5}$ reduction of Type IIB supergravity, although a full proof is still lacking. It is therefore expected that the above gravity-scalar theory should also be obtainable directly as a consistent truncation of eleven-dimensional or Type IIB supergravity. Indeed, the full non-linear ansatz for this reduction, valid for any $D$, was given in 14] and it was later proved in [15] that this is a consistent truncation of the higher-dimensional theory, that is, the equations of motion of the higher dimensional theory with the ansatz (2.13) are satisfied if and only if the equations of motion for the gravity-scalar system $(2.10)$ are satisfied in $D$ dimensions.

The reduction ansatz given in [14] is

$$
\begin{aligned}
d \hat{s}^{2} & =\Delta^{\frac{2}{D-1}} d s_{D}^{2}+\frac{4 l_{D}^{2}}{(D-3)^{2}} \Delta^{-\left(\frac{D-3}{D-1}\right)} \sum_{i=1}^{N} X_{i}^{-1} d \mu_{i}^{2} \\
\hat{F}_{(D)} & =\frac{(D-3)}{2 l_{D}} \sum_{i=1}^{N}\left(2 X_{i}^{2} \mu_{i}^{2}-\Delta X_{i}\right) \epsilon_{(D)}-\frac{l_{D}}{(D-3)} \sum_{i=1}^{N} X_{i}^{-1} *_{D} d X_{i} \wedge d\left(\mu_{i}^{2}\right),
\end{aligned}
$$

where

$$
\Delta=\sum_{i=1}^{N} X_{i} \mu_{i}^{2}
$$


and $\mu_{i}$ stand for a set of $N$ direction cosines satisfying

$$
\sum_{i=1}^{N} \mu_{i}^{2}=1 .
$$

Moreover, $\epsilon_{(D)}$ denotes the volume form of the metric $d s_{D}^{2}$, while the field strength $\hat{F}_{(D)}$ is identified with the M-theory four-form for $D=4$, its Hodge dual for $D=7$, and with the self-dual five-form of IIB supergravity for $D=5$.

\section{All asymptotically AdS Poincaré domain walls of the $\operatorname{SL}(N, \mathbb{R}) / \operatorname{SO}(N)$ sector}

From the discussion of the previous section we know that any solution of the equations of motion (2.10) in $D$ dimensions can be uplifted to solutions of either eleven-dimensional or Type IIB supergravity. In particular, any Poincaré domain wall of the form (1.1) corresponds to a solution of the higher-dimensional theory. Indeed, all supersymmetric asymptotically $A d S_{D}$ domain walls in $D=4,5,7$ have been constructed 18, 14, 5]. ${ }^{8}$ These domain walls solve the first order equations (1.7) with the true superpotential of the $\mathrm{SL}(N, \mathbb{R}) / \mathrm{SO}(N)$ sector of gauged maximal supergravity, which takes the form

$$
W_{o}=-\frac{(D-3)}{4 \kappa_{D}^{2} l_{D}} \sum_{i=1}^{N} X_{i} .
$$

It can be easily verified that this superpotential solves (1.8) with the scalar potential (2.8). The uplifted solutions are asymptotically $A d S_{4} \times S^{7}, A d S_{5} \times S^{5}$ or $A d S_{7} \times S^{4}$ and correspond to continuous distributions of parallel $M 2-, D 3-$ or $M 5$-branes respectively. Generically, they contain naked null singularities, corresponding to the location of the continuous brane distribution.

It was argued in [18, 14, 5], following [16, 17], that these supersymmetric solutions describe the RG flow of the dual CFTs due to the VEV of the scalar operators dual to the $\mathrm{SL}(N, \mathbb{R}) / \mathrm{SO}(N)$ scalars, with the VEVs defined by the brane distribution. Although in $D=5,7$ this interpretation is unique due to the unambiguous identification of the $\mathrm{SL}(N, \mathbb{R}) / \mathrm{SO}(N)$ scalars as dual to operators of dimension 2 and 4 respectively, in $D=4$ there is an ambiguity in the dimension of the operators dual to the $\mathrm{SL}(8, \mathbb{R}) / \mathrm{SO}(8)$ scalars. This because, as we will explain in detail below, the scalar potential (2.8) implies that the $\operatorname{SL}(N, \mathbb{R}) / \mathrm{SO}(N)$ scalars, except for $D=5$ in which case the mass saturates the Breitenlohner-Freedman (BF) bound [23], have a mass that allows their association with operators of two possible dimensions instead of one [24]. For $D=7$, however, this ambiguity is removed by symmetry. Namely, only the scalars of dimension 4 appear in the massless $\mathcal{N}=2$ supermultiplet. For $D=4$ the 35 dimension 1 scalars and the 35 dimension 2 scalars both appear in the massless $\mathcal{N}=8$ supermultiplet on an equal footing. Therefore, although the interpretation of these solutions in terms of VEVs for the dual operators

\footnotetext{
${ }^{8}$ The case $D=6$, corresponding to an $S^{4}$ reduction of massive Type IIA was also considered in 14$]$.
} 
remains correct, for $D=4$ there is a second possible interpretation in terms of deformations of the CFT Lagrangian. We will analyze this issue carefully below, when we compute in complete generality the VEVs of the possible dual operators.

In this section, however, we will try to systematically find all asymptotically AdS Poincaré domain wall solutions of the $\mathrm{SL}(N, \mathbb{R}) / \mathrm{SO}(N)$ sector, and in particular, all nonsupersymmetric ones. In other words, we will determine the most general fake superpotential $W(\phi)$ satisfying (1.8) with the scalar potential given by $(2.8) .{ }^{9}$ We will find that for $D=5$ and $D=7$, there are no analytic non-supersymmetric asymptotically AdS Poincaré domain walls. For $D=4$, however, we will show that there exists a continuum of analytic non-supersymmetric domain walls, continuously connected to the supersymmetric ones, as well as, a number of isolated non-supersymmetric domain walls.

Equation (1.8) is a first order non-linear PDE in $N-1$ variables for the fake superpotential $W(\phi)$, and as such, solving this equation in full generality for $W(\phi)$ seems a rather formidable task. However, not all solutions (1.8) are physically admissible, if one is interested in asymptotically AdS domain walls. In particular, the requirement that the domain walls defined by $W(\phi)$ via (1.7) asymptote to AdS space as $\phi^{I} \rightarrow 0$ implies that

$$
W(0)=-\frac{(d-1)}{\kappa_{D}^{2} l_{D}} .
$$

In addition we will assume that $W(\phi)$ admits a Taylor expansion around the maximally symmetric fixed point of $V(\phi)$ corresponding to $\phi^{I}=0$, namely

$$
W(\phi)=\sum_{n=0}^{\infty} W_{(n) I_{1} \ldots I_{n}} \phi^{I_{1}} \cdots \phi^{I_{n}},
$$

where all coefficients are completely symmetric in their indices, and $W_{(0)}=W(0)$ is given by (3.2). Within this framework, analyzing (1.8) in full generality is now tractable.

We start by Taylor expanding the scalar potential (2.8) around $\phi^{I}=0$. We find

$$
\begin{aligned}
V & =\sum_{n=0}^{\infty} V_{(n) I_{1} \ldots I_{n}} \phi^{I_{1}} \cdots \phi^{I_{n}} \\
& =-\frac{d(d-1)}{2 \kappa_{D}^{2} l_{D}^{2}}+\frac{1}{2} m_{I}^{2} \phi^{I} \phi^{I}-\frac{(d-2)(d-3) \sqrt{2} \kappa_{D}}{48 l_{D}^{2}} \sum_{i=1}^{N} b_{i I} b_{i J} b_{i K} \phi^{I} \phi^{J} \phi^{K}+\mathcal{O}\left(\phi^{4}\right),
\end{aligned}
$$

where $m_{I}^{2} l_{D}^{2}=\Delta_{I}\left(\Delta_{I}-d\right)=2(2-d)$. Inserting the expansions for $V(\phi)$ and $W(\phi)$ in (1.8) and matching powers one obtains the following recursion relations for the coefficients $W_{(n)}$ :

$$
\begin{aligned}
\sum_{m=0}^{n}[(m+1)(n-m+1) & \left.W_{(m+1)\left(I_{1} \ldots I_{m} J\right.} W_{(n-m+1)}{ }^{J} I_{m+1} \ldots I_{n}\right)_{s} \\
- & \left.\frac{d \kappa_{D}^{2}}{d-1} W_{(m)\left(I_{1} \ldots I_{m}\right.} W_{\left.(n-m) I_{m+1} \ldots I_{n}\right)_{s}}\right]=2 V_{(n)},
\end{aligned}
$$

\footnotetext{
${ }^{9}$ As we have mentioned already, the fake superpotential can in general be matrix valued, but we will only analyze the case of a scalar fake superpotential here.
} 
where $(\ldots)_{s}$ denotes symmetrization with weight 1 . For $n=0$ the recursion relations give

$$
W_{(1)} W_{(1)}^{J}-\frac{d \kappa_{D}^{2}}{d-1} W_{(0)}^{2}=2 V_{(0)} \text {. }
$$

Using the values for $W_{(0)}$ and $V_{(0)}$ from (3.2) and (3.4) we deduce that

$$
W_{(1) I}=0 .
$$

Since $V_{(1) I}=0$, which is guaranteed on general grounds by the requirement that AdS is a fixed point of the scalar potential, the equation for $n=1$, which reads

$$
\left(2 W_{(2) I J}+\frac{d}{l_{D}} \delta_{I J}\right) W_{(1)}^{J}=V_{(1) I},
$$

is automatically satisfied. Using the fact that $W_{(1) I}=0$, the next two equations now take the form

$$
\begin{aligned}
& n=2:\left(2 W_{(2) I J}+\frac{d}{l_{D}} \delta_{I J}\right) W_{(2)}{ }{ }_{K}=V_{(2) I K}, \\
& n=3:\left(6 W_{(2) I J}+\frac{d}{l_{D}} \delta_{I J}\right) W_{(3)}{ }^{J} K L=V_{(3) I K L},
\end{aligned}
$$

while for higher $n$ the recursion relations give

$$
\left(2 n W_{(2) I J}+\frac{d}{l_{D}} \delta_{I J}\right) W_{(n)}^{J} K_{1} \ldots K_{n-1}+\cdots=V_{(n) I K_{1} \ldots K_{n-1}},
$$

where the dots stand for terms involving the coefficients $W_{(m)}$ with $m<n$. It follows that, given the symmetric matrix $W_{(2) I J}$, the recursion relations uniquely determine all higher coefficients of $W(\phi)$, unless the matrix

$$
\left(2 n W_{(2) I J}+\frac{d}{l_{D}} \delta_{I J}\right)
$$

has some zero eigenvalues for some $n>2$. To address the question if and when this can happen we first have to solve equation (3.9) which determines $W_{(2) I J}$.

From (3.4) we see that $V_{(2) I J}=-\frac{(d-2)}{l_{D}^{2}} \delta_{I J}$. Since $W_{(2) I J}$ is a symmetric matrix it can be diagonalized by an orthogonal matrix $R_{I J}$. Such a (rigid) rotation in the space of the $N-1$ independent scalars would leave the form of the potential invariant since it simply rotates the weights $b_{i}$, while preserving the relations (2.6). Hence, we can take $W_{(2) I J}$ to be diagonal: $W_{(2) I J}=w_{I} \delta_{I J}$. Equation (3.9) then reduces to $N-1$ decoupled equations for the diagonal components, $w_{I}$, of $W_{(2) I J}$, namely

$$
\left(2 w_{I}+d / l_{D}\right) w_{I}+(d-2) / l_{D}^{2}=0, \quad I=1, \ldots, N-1,
$$

where there is no summation implied in this equation. The roots of this equation are $w_{I}=w_{ \pm}$, where $w_{+}=-1 / l_{D}, w_{-}=-(d-2) / 2 l_{D}$, and hence, for $d \neq 4$, there are $2^{N-1}$ independent solutions $W_{(2)}=\operatorname{diag}\left(w_{ \pm}, \ldots, w_{ \pm}\right)$, corresponding to the possible distributions of $w_{ \pm}$along the diagonal. For $d=4$ however, $w_{+}$and $w_{-}$coincide and there is a unique solution for $W_{(2)}$. It follows that the matrix (3.11) is diagonal with diagonal values 
$2 n w_{ \pm}+d / l_{D}$. Now, $2 n w_{+}+d / l_{D}=(d-2 n) / l_{D}$ can vanish if $d$ is even, i.e. $d=4,6$ since we are interested in the cases $d=3,4,6$. Similarly, $2 n w_{-}+d / l_{D}=(d-n(d-2)) / l_{D}$ can vanish if $d /(d-2)$ is integer, i.e. for $d=3,4$. However, in either case, $d=4$ requires $n=2$, which is excluded since we have already determined $W_{(2)}$. It follows that for $d=4$, the true superpotential $W_{o}$ given in (3.1) is the unique (physical) solution of (1.8). For $d=3,6$, however, we have seen that there are $2^{N-1}$ choices for $W_{(2)}$ and for each of them there is possibly some freedom in the value of $W_{(3)}$ due to the vanishing of some of the eigenvalues of the matrix (3.11), but all higher coefficients in $W(\phi)$ are completely determined once a choice for $W_{(2)}$ and $W_{(3)}$ has been made. Equation (3.9) however imposes further constraints. Noticing from (3.4) that $V_{(3)}$ vanishes for $d=3$ but not for $d>3$, equation (3.9) implies that for $d=6$, the matrix (3.11) must have no zero eigenvalues and therefore both $W_{(2)}=\operatorname{diag}\left(w_{-}, \ldots, w_{-}\right)$and $W_{(3)}$ are uniquely determined. So, as for $d=4, W_{o}$ in (3.1) is the unique solution of (1.8). For $d=3$, however, $V_{(3)}$ vanishes identically and so either the matrix (3.11) vanishes identically or $W_{(3)}$ vanishes identically. In the first case $W_{(2)}=\operatorname{diag}\left(w_{-}, \ldots, w_{-}\right)$and $W_{(3)}$ is arbitrary, while in the second case $W_{(3)}=0$ and $W_{(2)}$ can be any of the $2^{7}$ possible diagonal matrices. We conclude that $d=3$ is the only case which allows additional Poincare domain wall solutions beyond the supersymmetric ones corresponding to the superpotential (3.1). We will now examine these solutions more closely and construct explicitly as many of these as possible.

\section{The non-supersymmetric Poincaré domain walls in $D=4$}

As we have just shown, only in four dimensions $(d=3)$ are there physically acceptable solutions $W(\phi)$ to (1.8), in addition to the supersymmetric solution (3.1). These solutions fall into two general classes. The first case is when $W_{(3)}=0$ and $W_{(2)}=\operatorname{diag}\left(w_{ \pm}, \ldots, w_{ \pm}\right)$, where all signs are chosen independently. There are therefore $2^{7}$ such solutions corresponding to the different choices of the signs in $W_{(2)}$. However, since all seven scalars are equivalent, only 8 solutions are distinct, namely the ones corresponding to having $n+$ signs and $7-n-$ signs, with $n=0, \ldots, 7$. However, the solution where all signs are minus is covered by the second case, where $W_{(2)}=\operatorname{diag}\left(w_{-}, \ldots, w_{-}\right)$and $W_{(3)}$ is arbitrary. There are therefore only 7 distinct solutions in the first class. For the second class there is a unique choice for $W_{(2)}$, but $W_{(3)}$ is completely arbitrary. ${ }^{10}$ Since $W_{(3)}$ is a completely symmetric tensor of rank three, it has $\frac{1}{3 !}(N-1) N(N+1)=84$ independent components. There is therefore an 84-parameter family of solutions in this case. Note that this family is continuously connected to the supersymmetric solution corresponding to (3.1) since $W_{o}$ also has $W_{(2)}=\operatorname{diag}\left(w_{-}, \ldots, w_{-}\right)$. (See (6.10) below for the Taylor expansion of $W_{o}$.)

All these solutions can be constructed systematically using the recursion relations (3.5). However, obtaining the solutions in closed form by summing up the Taylor expansion is not very easy, if at all possible. We can, however, obtain in closed form a subclass of these solutions by going back to equation (1.8) and try to solve it exactly by first reducing the number of dynamical scalar fields in a way that is consistent with the equations of motion.

\footnotetext{
${ }^{10}$ Note however that restrictions on $W_{(3)}$ can arise as non-perturbative (in the scalar fields) effects. We will see how this happens in an exactly solvable case below.
} 


\begin{tabular}{|c|c|c|c|}
\hline$n$ & partition of 8 & scalar fields & isometry group \\
\hline 1 & 8 & 0 & $\mathrm{SO}(8)$ \\
\hline 2 & $1+7$ & 1 & $\mathrm{SO}(7)$ \\
& $2+6$ & & $\mathrm{SO}(2) \times \mathrm{SO}(6)$ \\
& $3+5$ & & $\mathrm{SO}(3) \times \mathrm{SO}(5)$ \\
& $4+4$ & 2 & $\mathrm{SO}(4) \times \mathrm{SO}(4)$ \\
\hline 3 & $1+1+6$ & & $\mathrm{SO}(6)$ \\
& $1+2+5$ & & $\mathrm{SO}(2) \times \mathrm{SO}(5)$ \\
& $1+3+4$ & & $\mathrm{SO}(3) \times \mathrm{SO}(4)$ \\
& $2+2+4$ & 3 & $\mathrm{SO}(2) \times \mathrm{SO}(2) \times \mathrm{SO}(4)$ \\
& $2+3+3$ & & $\mathrm{SO}(2) \times \mathrm{SO}(3) \times \mathrm{SO}(3)$ \\
\hline 4 & $1+1+1+5$ & & $\mathrm{SO}(5) \times \mathrm{SO}(4)$ \\
& $1+1+2+4$ & & $\mathrm{SO}(3) \times \mathrm{SO}(3)$ \\
& $1+1+3+3$ & & $\mathrm{SO}(2) \times \mathrm{SO}(2) \times \mathrm{SO}(3)$ \\
& $1+2+2+3$ & 4 & $\mathrm{SO}(2) \times \mathrm{SO}(2) \times \mathrm{SO}(2) \times \mathrm{SO}(2)$ \\
\hline 5 & $1+2+2+2$ & & $\mathrm{SO}(4)$ \\
& $1+1+1+2+3$ & 5 & $\mathrm{SO}(2) \times \mathrm{SO}(3)$ \\
& $1+1+2+2+2$ & & $\mathrm{SO}(2) \times \mathrm{SO}(2) \times \mathrm{SO}(2)$ \\
\hline 6 & $1+1+1+1+1+3$ & 5 & $\mathrm{SO}(3)$ \\
& $1+1+1+1+2+2$ & & $\mathrm{SO}(2) \times \mathrm{SO}(2)$ \\
\hline 7 & $1+1+1+1+1+1+1+2$ & 6 & - \\
\hline 8 & $1+1+1+1+1+1+1+1+1$ & 7 & \\
\hline
\end{tabular}

Table 1: The possible ways to reduce the number of dynamical scalar fields $X_{i}$, by setting a number of these equal to each other, correspond to the different partitions of 8 . The resulting isometry group is also shown.

A systematic way for doing this is setting some of the eight scalar fields $X_{i}$ equal to each other in all possible ways. Note that this is consistent with the equations of motion (2.10). The independent ways to set a number of the scalars $X_{i}$ equal is to consider all possible $n$-partitions of 8 . Each $n$-partition corresponds to an independent way to keep $n-1$ dynamical scalar fields. Table 1 lists all such partitions, together with the corresponding isometry group [5]. We will attempt to find a closed form for the above solutions only for the cases with a single dynamical field, however. As we will see, even this seemingly innocuous case, requires considerable effort.

\subsection{Domain walls with a single scalar}

The four distinct one-scalar truncations in table 1 are obtained by setting $X_{1}=\ldots=$ $X_{k} \equiv X, X_{k+1}=\ldots=X_{8}=X^{-k /(8-k)}$, where $k=4,5,6,7$. In this section we will keep $k$ as a parameter, however, so that we can discuss all four cases simultaneously. The scalar 


\begin{tabular}{|c|c|c|c|}
\hline $\mathrm{k}$ & isometry group & $-16 \kappa^{2} l^{2} V$ & fixed points \\
\hline 7 & $\mathrm{SO}(7)$ & $35 X^{2}+14 X^{-6}-X^{-14}$ & $X=1,1 / 5^{1 / 8}$ \\
6 & $\mathrm{SO}(2) \times \mathrm{SO}(6)$ & $24\left(X^{2}+X^{-2}\right)$ & $X=1$ (double) \\
5 & $\mathrm{SO}(3) \times \mathrm{SO}(5)$ & $3\left(5 X^{2}+X^{-10 / 3}+10 X^{-2 / 3}\right)$ & $X=1$ \\
4 & $\mathrm{SO}(4) \times \mathrm{SO}(4)$ & $8\left(X^{2}+X^{-2}+4\right)$ & $X=1$ (double) \\
\hline
\end{tabular}

Table 2: The scalar potential for the four possible one-scalar truncations. Note that the fixed point $X=1$, common to all potentials, corresponds to the AdS fixed point at $\phi=0$.

potential (2.8), which for general $k$ takes the form ${ }^{11}$

$$
V=-\frac{1}{16 \kappa^{2} l^{2}}\left(k(k-2) X^{2}+2 k(8-k) X^{-2(k-4) /(8-k)}+(8-k)(6-k) X^{-2 k /(8-k)}\right),
$$

is shown explicitly for each of the four cases in table 2. It is useful to parameterize the single scalar field $X$ in terms of a scalar with a canonical kinetic term as

$$
X=e^{\sqrt{\frac{8-k}{2 k}} \kappa \phi} .
$$

Equation then (1.8) takes the form

$$
V=\frac{\kappa^{2}}{4}\left(\frac{(8-k)}{k}\left(X \partial_{X} W\right)^{2}-3 W^{2}\right)
$$

Moreover, the superpotential (3.1) becomes

$$
W_{o}=-\frac{1}{4 \kappa^{2} l}\left(k X+(8-k) X^{-\frac{k}{(8-k)}}\right),
$$

and it is easily seen to be a solution of (4.3).

We have seen above that there exists a one-parameter family of functions $W(\phi ; \alpha)$ which contains $W_{o}(\phi)$ as a special case. In particular, the Taylor expansions of $W(\phi ; \alpha)$ around $\phi=0$, for a generic value of the free parameter $\alpha$, and of $W_{o}(\phi)$ have the same quadratic term, corresponding to $w_{-}$in the notation of the previous section. ${ }^{12}$ In addition, however, there exists another isolated solution, $\widetilde{W}_{o}(\phi)$, whose quadratic term corresponds to $w_{+}$and whose cubic term vanishes. The Taylor expansions of $W(\phi ; \alpha)$ and $\widetilde{W}_{o}(\phi)$ around $\phi=0$ are therefore not continuously connected. This though does not exclude the possibility that, non-perturbatively in $\phi, W(\phi ; \alpha)$ and $\widetilde{W}_{o}(\phi)$ are continuously connected. Remarkably, we will see below in an example where the exact one-parameter family $W(\phi ; \alpha)$

\footnotetext{
${ }^{11}$ From now on we drop the subscript $D$ in the gravitational constant $\kappa$ and the AdS radius $l$ since we will always work in $D=4$.

${ }^{12}$ Recall that the parameter $\alpha$ first enters in the cubic term in the Taylor expansion around $\phi=0$.
} 
can be obtained exactly that $W(\phi ; \alpha)$ interpolates between the supersymmetric solution $W_{o}(\phi)$ and $\widetilde{W}_{o}(\phi)$.

In the next section we will address systematically the problem of solving equation (4.3) exactly. For the moment, however, we can use the fact that $W(\phi ; \alpha)$ is continuously connected to $W_{o}(\phi)$ in order to obtain $W(\phi ; \alpha)$ in an expansion in the free parameter $\alpha$, for general $k$. Obviously, this approach can provide no information on $\widetilde{W}_{o}(\phi)$. We start by writing $W(\phi ; \alpha)$ in a formal asymptotic expansion $\operatorname{as}^{13}$

$$
W(\phi ; \alpha)=W_{o}(\phi)+\sum_{n=1}^{\infty}\left(\frac{-1}{32 \kappa^{2} l}\right)^{n}\left(\alpha-\alpha_{o}\right)^{n} W^{(n)}(\phi),
$$

where, $\alpha_{o}=-(8-k)(k-4) k / 24$ and $W\left(\phi ; \alpha_{o}\right) \equiv W_{o}(\phi)$. Inserting this expansion into (4.3) one obtains an infinite set of linear equations for the functions $W^{(n)}(\phi)$, namely

$$
\begin{array}{r}
\left(X \partial_{X} W_{o} X \partial_{X}-\frac{3 k}{(8-k)} W_{o}\right) W^{(n)} \\
+\frac{1}{2} \sum_{m=1}^{n-1}\left(X \partial_{X} W^{(m)} X \partial_{X} W^{(n-m)}-\frac{3 k}{(8-k)} W^{(m)} W^{(n-m)}\right)=0
\end{array}
$$

which can be solved iteratively. For $n=1$, this equation is homogeneous and its solution is

$$
W^{(1)}=\left(\frac{X^{\frac{8}{(8-k)}}-1}{X}\right)^{3}
$$

Note that, as expected from the general analysis above, $W^{(1)}=\mathcal{O}\left(\phi^{3}\right)$ as $\phi \rightarrow 0$. For $n>1$ equation (4.6) is non-homogeneous but it can be solved with the help of an integrating factor

$$
R=\exp \left(-\frac{3 k}{(8-k)} \int \frac{d X}{X^{2}} \frac{W_{o}}{\partial_{X} W_{o}}\right)=\frac{1}{W^{(1)}} .
$$

The solution then takes the form

$$
W^{(n)}=W^{(1)} \int \frac{d X}{X} \frac{Q_{n}(X)}{W^{(1)}(X)}+c_{n} W^{(1)}
$$

where $c_{n}$ are constants and

$$
Q_{n}=-\frac{1}{2 X \partial_{X} W_{o}} \sum_{m=1}^{n-1}\left(X \partial_{X} W^{(m)} X \partial_{X} W^{(n-m)}-\frac{3 k}{(8-k)} W^{(m)} W^{(n-m)}\right) .
$$

In particular,

$$
W^{(2)}=6 \kappa^{2} l\left(\frac{X^{\frac{8}{(8-k)}}-1}{X}\right)^{3}\left(\frac{1}{(8-k)} X^{\frac{4(k-2)}{(8-k)}}+\frac{2}{(k-4)}\left(X^{\frac{4(k-4)}{(8-k)}}-1\right)+\frac{1}{k} X^{\frac{4(k-6)}{(8-k)}}+c_{2}(k)\right),
$$

\footnotetext{
${ }^{13}$ The normalization of the free parameter is chosen so that it matches the natural free parameter of the exact solution that we will present in the next section for $k=6$.
} 
where the term involving $k-4$ in the denominator is understood as the limit $k \rightarrow 4$, giving $\log X$, for the case $k=4$. Moreover, the constant $c_{2}(k)$ is not arbitrary. It is uniquely fixed by the requirement that $W^{(2)}$ does not contribute to the cubic term in $\phi$ of $W(\phi ; \alpha)$, which is necessary in order to identify $\left(\alpha-\alpha_{o}\right)$ (as opposed to some other function of $\alpha$ ) with the free parameter of $W$.

$$
c_{2}(k)=-\frac{8}{k(8-k)} .
$$

The same argument determines all constants $c_{n}$ in $Q_{n}$. Putting everything together, to this order we have

$$
\begin{aligned}
W(\phi ; \alpha)= & -\frac{1}{4 \kappa^{2} l}\left(k X+(8-k) X^{-\frac{k}{(8-k)}}\right) \\
& -\frac{1}{32 \kappa^{2} l}\left(\alpha-\alpha_{o}\right)\left(\frac{X^{\frac{8}{(8-k)}}-1}{X}\right)^{3}\left\{1-\frac{3}{16}\left(\alpha-\alpha_{o}\right)\left(\frac{1}{(8-k)} X^{\frac{4(k-2)}{(8-k)}}\right.\right. \\
& \left.\left.+\frac{2}{(k-4)}\left(X^{\frac{4(k-4)}{(8-k)}}-1\right)+\frac{1}{k} X^{\frac{4(k-6)}{(8-k)}}-\frac{8}{k(8-k)}\right)\right\}+\mathcal{O}\left(\left(\alpha-\alpha_{o}\right)^{3}\right) .
\end{aligned}
$$

Given this perturbative (in $\alpha-\alpha_{o}$ ) fake superpotential, we can immediately obtain the corresponding domain wall solutions via the first order equations (1.7). We give explicitly the form of these backgrounds to first order in $\alpha-\alpha_{o}$ in appendix $\mathbb{A}$, since we will need them for the computation of the one- and two-point functions of the field theory duals of these domain walls.

\subsection{Exact closed form solutions}

Having obtained a perturbative solution for $W(\phi ; \alpha)$ for all possible values of $k$, let us now try to solve (4.3) exactly. This should determine not only the full $W(\phi ; \alpha)$, but also $\widetilde{W}_{o}(\phi)$. It was observed in [12] that for a single scalar field, $\phi$, equation (1.8), with an arbitrary potential, can be recast in a standard form by means of the field redefinitions

$$
\psi=\sqrt{\frac{d \kappa^{2}}{d-1}} \phi, \quad y=\operatorname{coth}(u), \quad W=l v \cosh (u)
$$

where

$$
v=-\left(-\frac{2(d-1)}{d \kappa^{2} l^{2}} V\right)^{1 / 2}
$$

In terms of these variables, equation (1.8) takes the form ${ }^{14}$

$$
y^{\prime}(\psi)=\left(\frac{v^{\prime}}{v} y-1\right)\left(y^{2}-1\right)
$$

where the prime denotes derivative with respect to $\psi$. This equation is a special case of Abel's equation of the first kind [25]

$$
y^{\prime}=f_{3}(\psi) y^{3}+f_{2}(\psi) y^{2}+f_{1}(\psi) y+f_{0}(\psi),
$$

\footnotetext{
${ }^{14}$ Note that the obvious solutions $y= \pm 1$ of this equation are rejected since, via (4.14), they correspond to $u \rightarrow \infty$ and hence $W \rightarrow \infty$.
} 
where $f_{i}(\psi)$ are arbitrary functions. Abel's equation can in turn be cast in the canonical form

$$
z^{\prime}=\tilde{f}_{3}(\psi) z^{3}+\tilde{f}_{1}(\psi) z+\tilde{f}_{0}(\psi)
$$

by means of the transformation

$$
y=z-\frac{f_{2}}{3 f_{3}} .
$$

Clearly, equation (4.18) can be integrated directly if either $\tilde{f}_{3}$ or $\tilde{f}_{0}$ vanish. Moreover, it can also be integrated directly if 'Abel's invariant'

$$
\mathcal{I} \equiv-\frac{\left(\tilde{f}_{0} \tilde{f}_{3}^{\prime}-\tilde{f}_{0}^{\prime} \tilde{f}_{3}+3 \tilde{f}_{0} \tilde{f}_{3} \tilde{f}_{1}\right)^{3}}{27 \tilde{f}_{3}^{4} \tilde{f}_{0}^{5}}
$$

is a constant 25]. If it is not a constant, however, no general solution of (4.18) is known. In that case one can only hope that the equation at hand falls into one of the known integrable classes of Abel's equation, each of which has a very particular way of solution that is not applicable to other classes. Some recent investigations and overviews of Abel's equation and its known integrable classes can be found in [26, 25].

In our case, however, the functions $f_{i}(\psi)$ are not completely arbitrary since they are all related to the scalar potential. Specifically, from (4.16) we read

$$
f_{3}=-f_{1}=q^{\prime}, \quad f_{2}=-f_{0}=-1,
$$

where $q \equiv \log |v|$. Moreover, one can easily compute the tilded coefficients corresponding to the transformed equation (4.18):

$$
\tilde{f}_{3}=q^{\prime}, \quad \tilde{f}_{1}=-\frac{1}{3 q^{\prime}}\left(1+3 q^{2}\right), \quad \tilde{f}_{0}=\frac{1}{3 q^{\prime 2}}\left(q^{\prime \prime}+2 q^{\prime 2}-2 / 9\right) .
$$

It follows that for a generic potential, and hence a generic $q$, Abel's invariant is not automatically constant. Requiring that it be a constant, leads to a second order, non-linear differential equation for $q^{\prime}$, which seems more difficult to solve than the original first order equation. However, as we have already pointed out, requiring that either $\tilde{f}_{3}$ or $\tilde{f}_{0}$ vanish, also leads to a solvable equation. These conditions lead to differential equations for the potential, which are easily solvable. In particular, $\tilde{f}_{3}=q^{\prime}=0$ gives the constant potential

$$
V=-\frac{d(d-1)}{2 \kappa^{2} l^{2}}
$$

corresponding to exact AdS space. More interesting is the condition $\tilde{f}_{0}=\frac{1}{3 q^{\prime 2}}\left(q^{\prime \prime}+2 q^{\prime 2}-\right.$ $2 / 9)=0$, which leads to the potential

$$
V=-\frac{d(d-1)}{2 \kappa^{2} l^{2}} \cosh \left(\frac{2 \psi}{3}\right)
$$

The observation that this potential leads to a soluble Abel's equation was the only motivation for considering this potential in [12]. Curiously, however, noting from (4.2) and (4.14) that, for $d=3, X$ and $\psi$ are related by

$$
X=e^{\sqrt{\frac{8-k}{3 k}} \psi},
$$


the potential (4.24) is seen to be identical to the potential (4.1) for $k=6$. Hence, at least for the case $k=6$, we are able to solve (4.3) exactly. However, the potential (4.1) with generic $k$ gives

$$
q^{\prime}=\frac{v^{\prime}}{v}=\sqrt{\frac{k(8-k)}{3}}\left(\frac{(k-2) e^{16 \psi / \sqrt{3 k(8-k)}}-2(k-4) e^{8 \psi / \sqrt{3 k(8-k)}}-(6-k)}{(k-2) e^{16 \psi / \sqrt{3 k(8-k)}}+2(8-k) e^{8 \psi / \sqrt{3 k(8-k)}}+(6-k)(8-k)}\right) .
$$

One can now easily check that, except for $k=6$ in which case $\tilde{f}_{0}$ vanishes, Abel's invariant is not constant for any value of $k$. As we discussed already, this makes it much harder to solve (4.16) for the potential (4.1) with $k \neq 6$.

To obtain the exact solution for the case $k=6$, we start by inserting the potential (4.24) in equation (4.16). The resulting equation takes the form

$$
\frac{2}{1-s^{2}} \frac{d s}{d \rho}+\frac{1}{1-\rho^{2}}\left(\frac{\rho}{s}-3\right)=0
$$

where

$$
s=\frac{1}{y}, \quad \rho=\tanh \left(\frac{2 \psi}{3}\right) .
$$

The general solution of this equation is 12

$$
s=\frac{\rho}{1 \pm\left(1-\rho^{2}\right)\left(1+2 \alpha \rho+\rho^{2}\right)^{-1 / 2}},
$$

where $\alpha$ is an integration constant. Since the conformal boundary corresponds to $\rho=0$, we can take $\rho \geq 0$. The choice $\rho \leq 0$ is also possible but it is equivalent. The value of the integration constant $\alpha$ is then restricted by the requirement that $1+2 \alpha \rho+\rho^{2} \geq 0$. This is guaranteed provided

$$
\alpha \geq-1
$$

The fake superpotential is now obtained from (4.14) as

$$
W(\phi ; \alpha)=-\frac{2}{\kappa^{2} l} \frac{1}{\left(1-\rho^{2}\right)^{1 / 4}} \frac{1}{\sqrt{1-s^{2}}} .
$$

Expanding this for small $\psi$, we see that the solution with the negative sign in (4.29) always contains a linear term in $\psi$ and it is therefore rejected. For the positive sign solution we find

$$
W(\phi ; \alpha)=-\frac{2}{\kappa^{2} l}\left(1+\frac{1}{6} \psi^{2}+\frac{1}{27} \alpha \psi^{3}+\mathcal{O}\left(\psi^{4}\right)\right),
$$

which is precisely of the required form. We therefore expect that this is the full oneparameter family of fake superpotentials whose existence we predicted above on general grounds and which we computed perturbatively in the free parameter. In particular, it should contain the true superpotential (4.4), which for $k=6$ becomes

$$
W_{o}(\phi)=-\frac{1}{2 \kappa^{2} l}\left(3 e^{\psi / 3}+e^{-\psi}\right) .
$$


Indeed, this is the case as it is easy to check that for $\alpha=-1, W(\phi ; \alpha)$ reduces to $W_{o}(\phi)$ :

$$
W(\phi ;-1)=W_{o}(\phi)
$$

Since $W(\phi ; \alpha)$ is the most general solution, however, one wonders where is the solution $\widetilde{W}_{o}(\phi)$ which we have predicted and whose expansion around $\phi=0$ should have a different quadratic term from that of $W(\phi ; \alpha)$. The answer is that $\widetilde{W}_{o}(\phi)$ is obtained from $W(\phi ; \alpha)$ by sending $\alpha$ to infinity:

$$
\widetilde{W}_{o}(\phi)=\lim _{\alpha \rightarrow \infty} W(\phi ; \alpha)=-\frac{2}{\kappa^{2} l} \cosh ^{3 / 2}\left(\frac{2 \psi}{3}\right) .
$$

Expanding this for small $\psi$ we find

$$
\widetilde{W}_{o}(\phi)=-\frac{2}{\kappa^{2} l}\left(1+\frac{1}{3} \psi^{2}+\mathcal{O}\left(\psi^{4}\right)\right) .
$$

This has precisely the desired form, namely a quadratic term corresponding to $w_{+}$and a vanishing cubic term. The fake superpotential $W(\phi ; \alpha)$, therefore, interpolates between the supersymmetric superpotential $W_{o}(\phi)=W(\phi ;-1)$ and $\widetilde{W}_{o}(\phi)=W(\phi ; \infty)$.

\section{Exact non-supersymmetric membrane flows}

All non-supersymmetric domain wall solutions we have obtained above in $D=4$, in closed form or not, can in principle be uplifted to asymptotically $A d S_{4} \times S^{7}$ non-supersymmetric solutions of eleven-dimensional supergravity using the ansatz (2.13). We will only uplift explicitly the closed form solutions we found in the previous section, however. To do this we first need to determine the four-dimensional domain wall metrics corresponding to the exact fake superpotentials for $k=6$.

Integrating the first order equations (1.7) using the fake superpotential (4.31) we find that the full one-parameter family of Poincaré domain walls takes the form

$$
\begin{aligned}
d s_{\alpha}^{2} & =\frac{1}{2 \rho^{2}}\left(1+\alpha \rho+\sqrt{1+2 \alpha \rho+\rho^{2}}\right)\left(\frac{l^{2} d \rho^{2}}{\sqrt{1-\rho^{2}}\left(1+2 \alpha \rho+\rho^{2}\right)}+\xi^{2} \sqrt{1-\rho^{2}} \eta_{i j} d x^{i} d x^{j}\right) \\
\phi & =\sqrt{\frac{3}{2 \kappa^{2}}} \tanh ^{-1} \rho .
\end{aligned}
$$

The integration constant $\xi^{2}$ can be absorbed by a rescaling of the transverse coordinates $x^{i}$, but we have introduced it for reasons that will become clear soon. Namely, for any finite value of $\alpha$, and taking $\xi^{2}=1$, the metric (5.1) is asymptotically AdS with canonical radial coordinate $\rho \sim e^{-r / l}$ as $\rho \rightarrow 0$. In particular, the supersymmetric metric corresponding to $\alpha=-1$ reads

$$
d s_{-1}^{2}=\frac{1}{\rho^{2}}\left(\frac{l^{2} d \rho^{2}}{\sqrt{1+\rho}(1-\rho)^{3 / 2}}+\sqrt{1+\rho}(1-\rho)^{3 / 2} \eta_{i j} d x^{i} d x^{j}\right) .
$$


In order for the metric (5.1) to have a well-defined limit as $\alpha \rightarrow \infty$, however, we must take $\xi^{2} \sim$ const. $/ \alpha$ as $\alpha \rightarrow \infty$. Taking $\xi^{2} \sim 2 / \alpha$ and evaluating the limit $\alpha \rightarrow \infty$, (5.1) becomes

$$
d s_{\infty}^{2}=\frac{l^{2} d \rho^{2}}{4 \rho^{2} \sqrt{1-\rho^{2}}}+\frac{\sqrt{1-\rho^{2}}}{\rho} \eta_{i j} d x^{i} d x^{j} .
$$

This is again an asymptotically AdS metric, but with canonical radial coordinate $\sqrt{\rho} \sim$ $e^{-r / l}$.

Note that these metrics are non-singular for $0 \leq \rho<1$. There is a singularity at $\rho=1$, however, which is in fact a curvature singularity from the four-dimensional point of view and the scalar field also diverges at this point. In fact, the curvature singularity of the supersymmetric metric, for which the Ricci scalar behaves like $R_{4} \sim(1-\rho)^{-1 / 2}$ as $\rho \rightarrow 1$, is milder that the curvature singularity of the non-supersymmetric metrics, for which $R_{4} \sim(1-\rho)^{-3 / 2}$ as $\rho \rightarrow 1$. Moreover, the singularity is null for the supersymmetric case but timelike for the non-supersymmetric metric [11]. Nevertheless, in both cases the singularity is 'good' according to the criterion of [11] since the scalar potential (4.24) is bounded from above, not only on-shell but even off-shell. Accordingly, in both cases, the presence of the singularity signals some genuine IR phenomenon in the dual field theory.

We can now use the ansatz (2.13) to uplift the four-dimensional solution (5.1) to eleven dimensions. It is convenient, however, to first use a reduced ansatz obtained from (2.13) by setting the 8 scalars $X_{i}$ pairwise equal [14]:

$$
X_{2 a-1}=X_{2 a} \equiv \tilde{X}_{a}, \quad a=1,2,3,4,
$$

so that $\tilde{X}_{1} \tilde{X}_{2} \tilde{X}_{3} \tilde{X}_{4}=1$. This reduction corresponds to the scalar sector of the truncation of $\mathcal{N}=8$ supergravity to the maximal abelian subgroup, $\mathrm{U}(1)^{4}$, of its gauge group $\mathrm{SO}(8)[27,28] .{ }^{15}$ Note, that this reduction does not include all possible one-scalar truncations discussed in section 4.1 since the cases $k=3,5$ are not consistent with this reduction. It does cover however the cases $k=4$ and $k=6$, which is the case we are interested here. The reduced ansatz reads

$$
\begin{aligned}
& d \hat{s}_{11}^{2}=\tilde{\Delta}^{2 / 3} d s_{4}^{2}+4 l^{2} \tilde{\Delta}^{-1 / 3} \sum_{a=1}^{4} \tilde{X}_{a}^{-1}\left(d \tilde{\mu}_{a}^{2}+\tilde{\mu}_{a}^{2} d \phi_{a}^{2}\right), \\
& \hat{F}_{(4)}=\frac{1}{l} \sum_{a=1}^{4}\left(\tilde{X}_{a}^{2} \tilde{\mu}_{a}^{2}-\tilde{\Delta} \tilde{X}_{a}\right) \epsilon_{(4)}-l \sum_{a=1}^{4} \tilde{X}_{a}^{-1} * d \tilde{X}_{a} \wedge d\left(\tilde{\mu}_{a}^{2}\right),
\end{aligned}
$$

where $\tilde{\Delta}=\sum_{a=1}^{4} \tilde{X}_{a} \tilde{\mu}_{a}^{2}$ and the quantities $\tilde{\mu}_{a}$ and the four angles $\phi_{a}, 0 \leq \phi_{a} \leq 2 \pi$, are related to the direction cosines $\mu_{i}$ in 2.13 by

$$
\mu_{2 a-1}=\tilde{\mu}_{a} \cos \phi_{a}, \quad \mu_{2 a}=\tilde{\mu}_{a} \sin \phi_{a}, \quad a=1,2,3,4,
$$

so that $\sum_{a=1}^{4} \tilde{\mu}_{a}^{2}=\sum_{i=1}^{8} \mu_{i}^{2}=1$. The four $\tilde{\mu}_{a}$ can be parameterized in terms of the angles on a three-sphere as

$$
\tilde{\mu}_{1}=\cos \theta \cos \chi \cos \omega, \quad \tilde{\mu}_{2}=\cos \theta \cos \chi \sin \omega, \quad \tilde{\mu}_{3}=\cos \theta \sin \chi, \quad \tilde{\mu}_{4}=\sin \theta,
$$

\footnotetext{
${ }^{15}$ The $\mathrm{U}(1)$ gauge fields and the three axions are set to zero here, however.
} 
$0 \leq \theta, \chi \leq \pi, 0 \leq \omega \leq 2 \pi$. Finally, the four scalars $\tilde{X}_{a}$ can be parameterized in terms of three dilatonic scalars $\overrightarrow{\tilde{\varphi}}=\left(\tilde{\varphi}_{1}, \tilde{\varphi}_{2}, \tilde{\varphi}_{3}\right)$ :

$$
\tilde{X}_{a}=e^{-\frac{1}{2} \overrightarrow{\tilde{b}} \cdot \overrightarrow{\tilde{\varphi}}}
$$

where $\overrightarrow{\tilde{b}}_{i}$ satisfy

$$
\overrightarrow{\tilde{b}}_{a} \cdot \overrightarrow{\tilde{b}}_{b}=4 \delta_{a b}-1
$$

A convenient choice for $\overrightarrow{\tilde{b}}_{a}$ is

$$
\overrightarrow{\tilde{b}}_{1}=(1,-1,-1), \quad \overrightarrow{\tilde{b}}_{2}=(-1,1,-1), \quad \overrightarrow{\tilde{b}}_{3}=(-1,-1,1), \quad \overrightarrow{\tilde{b}}_{4}=(1,1,1) .
$$

The $k=6$ solution now corresponds to setting $\tilde{X}_{1}=\tilde{X}_{2}=\tilde{X}_{3} \equiv X, \tilde{X}_{4}=X^{-3}$. Recalling from (4.25) that $X=e^{\psi / 3}$ and the relation between $\psi$ and $\rho$ from (4.28), we deduce that

$$
X=\left(\frac{1+\rho}{1-\rho}\right)^{1 / 4}
$$

Hence,

$$
\tilde{\Delta}=\left(\frac{1+\rho}{1-\rho}\right)^{1 / 4} \cos ^{2} \theta+\left(\frac{1-\rho}{1+\rho}\right)^{3 / 4} \sin ^{2} \theta .
$$

Putting everything together, we have the following two solutions of eleven-dimensional supergravity

$$
\begin{aligned}
d \hat{s}_{11}^{2}=\tilde{\Delta}^{2 / 3} d s_{4}^{2}+4 l^{2} \tilde{\Delta}^{-1 / 3}\{( & \left.\frac{1+\rho}{1-\rho}\right)^{3 / 4}\left[\left(\cos ^{2} \theta+\left(\frac{1-\rho}{1+\rho}\right) \sin ^{2} \theta\right) d \theta^{2}+\sin ^{2} \theta d \phi_{4}^{2}\right] \\
& \left.+\left(\frac{1-\rho}{1+\rho}\right)^{1 / 4} \cos ^{2} \theta d \Omega_{5}^{2}\right\}
\end{aligned}
$$

where $d s_{4}^{2}$ is given either by the finite- $\alpha$ metric (5.1) or by the $\alpha \rightarrow \infty$ metric (5.3). Correspondingly, the four-form field strength is given by

$$
\begin{aligned}
\hat{F}_{(4)}^{\alpha}= & \frac{\left(1+\alpha \rho+\sqrt{1+2 \alpha \rho+\rho^{2}}\right)}{2 \rho^{2} \sqrt{1+2 \alpha \rho+\rho^{2}}} \\
& \left\{\frac{(1+\rho)}{2 \rho^{2}}\left[2 \cos ^{2} \theta+\left(\frac{1-\rho}{1+\rho}\right)\left(1+2 \sin ^{2} \theta\right)\right]\left(1+\alpha \rho+\sqrt{1+2 \alpha \rho+\rho^{2}}\right) d \rho\right. \\
& \left.+4\left(1+2 \alpha \rho+\rho^{2}\right) \cos \theta \sin \theta d \theta\right\} \wedge \bar{\epsilon}_{(3)}, \\
\hat{F}_{(4)}^{\infty}= & \frac{1}{\sqrt{\rho}}\left\{\frac{(1+\rho)}{2 \rho^{2}}\left[2 \cos ^{2} \theta+\left(\frac{1-\rho}{1+\rho}\right)\left(1+2 \sin ^{2} \theta\right)\right] d \rho+8 \cos \theta \sin \theta d \theta\right\} \wedge \bar{\epsilon}_{(3)} .
\end{aligned}
$$

It is not difficult to check that these satisfy $\mathrm{d} \hat{F}_{(4)}=0$ and $\mathrm{d} \hat{*} \hat{F}_{(4)}=0$. Of course, (5.14) and (5.15)- 5.16) also satisfy Einstein's equation in eleven dimensions, as is guaranteed by 
the fact that the four-dimensional theory is a consistent truncation of eleven-dimensional supergravity [15]. We have not checked this explicitly, however.

A few comments are in order here. First, note that the compact part the metric (5.14) does not depend on the parameter $\alpha$ and hence it describes the same inhomogeneous deformation of $S^{7}$ as the supersymmetric solution with $\alpha=-1$. Namely, at $\rho=0$ the compact part of the metric is exactly the metric on $S^{7}$. As one moves away from $\rho=0$ the $S^{7}$ is deformed to a warped product of an $S^{5}$ of decreasing radius and a squashed $S^{2}$ with increasing radius. At $\rho=1$, the $S^{5}$ shrinks to zero size, while the $S^{2}$ becomes totally squashed, but with infinite radius. The supersymmetric solution corresponds to a continuous non-uniform distribution of $M 2$-branes on a disc of finite radius on the equatorial plane of the squashed $S^{2}$ [16, 14]. It would be very interesting to find an analogous interpretation for the non-supersymmetric solutions. As the four-dimensional solutions, the uplifted metrics have a curvature singularity at $\rho=1$, but now the eleven-dimensional Ricci scalar behaves like $\hat{R}=\frac{1}{6} \hat{F}_{(4)}{ }^{2} \sim(1-\rho)^{-1 / 3}$ as $\rho \rightarrow 1$, independently of the value of $\alpha$. Of course, the singularity remains null for the supersymmetric case and timelike for the non-supersymmetric one since the uplift does not alter the causal structure. However, at least for the supersymmetric solution, the uplift helps identify the cause of the singularity, namely the fact that the distribution of the $M 2$-branes is continuous [16], and as a result understand how M-theory resolves the singularity. A similar interpretation for the non-supersymmetric solution would therefore clarify the nature of the singularity. Another important difference between the supersymmetric and non-supersymmetric solutions is that the part of the metric orthogonal to the squashed $S^{2}$ becomes conformal to $A d S_{4} \times S^{5}$ as $\rho \rightarrow 0$ for the supersymmetric case, while for the non-supersymmetric case it becomes conformal to $\mathbb{R}^{4} \times S^{5}$ as $\rho \rightarrow 0$. Moreover, $\hat{F}_{(4)}$ vanishes at $\rho=0$ for the supersymmetric case, while it is finite but non-zero for the non-supersymmetric one.

Interestingly, the same scalar potential (4.24), corresponding to $k=6$, led to the MTZ black hole in four dimensions [19]. Since we know how to uplift solutions of the four-dimensional scalar-gravity system with this potential to eleven dimensions, we find it tempting to present the eleven-dimensional black hole metric explicitly, which we do in appendix B.

\section{Holographic one-point functions}

The asymptotically AdS domain walls (1.1) describe, via the AdS/CFT duality, the RG flow of the field theory living on the conformal boundary. Such an RG flow can result from a deformation of the Lagrangian of the UV CFT by a relevant operator, or from a non-conformal vacuum, described by the VEVs of certain operators. To determine which of these possibilities is realized in a given domain wall background, one should evaluate holographically the one-point functions of the operators dual to the non-trivial scalar fields, as well as the one-point function of the stress tensor.

One, therefore, first needs to identify the gauge-invariant operator $\mathcal{O}_{\Delta}$ dual to a given scalar field. Recall that the mass, $m$, of a scalar field is related to the dimension, $\Delta$, of the 
dual operator via

$$
m^{2} l^{2}=\Delta(\Delta-d)
$$

Since this equation has two roots, $\Delta_{ \pm}$, the question arises as to which of the two is the dimension of the dual operator. It was argued in [24] that while for $m^{2} l^{2}>-(d / 2)^{2}+1$ the dual operator must unambiguously have dimension $\Delta_{+}$, for

$$
-\left(\frac{d}{2}\right)^{2} \leq m^{2} l^{2} \leq-\left(\frac{d}{2}\right)^{2}+1
$$

both $\Delta_{ \pm}$are possible dimensions for the dual operator. More specifically, there are two possible quantizations of the scalar field, corresponding to the two dimensions $\Delta_{ \pm}$of the dual operator [23]. The resulting generating functionals of correlation functions of the corresponding operators are then related by a Legendre transformation as we will review below.

An important property of the $\mathrm{SL}(N, \mathbb{R}) / \mathrm{SO}(N)$ scalars is that their mass falls precisely in the range (6.2) allowing two quantizations. Namely, recall from (3.4) that the mass of the scalar fields of the $\mathrm{SL}(N, \mathbb{R}) / \mathrm{SO}(N)$ sector is

$$
m_{I}^{2} l_{D}^{2}=2(2-d)
$$

With this mass, the condition (6.2) translates into ${ }^{16}$

$$
2 \leq d \leq 6
$$

which includes all cases for $d$ we are interested in, namely $d=3,4,6$. The two possible dimensions are

$$
\Delta_{ \pm}=\frac{d}{2} \pm \frac{1}{2}|d-4|,
$$

which coincide for $d=4$. In this case the mass saturates the BF bound $m^{2} l^{2} \geq-(d / 2)^{2}$, and there is a unique quantization [24]. For $d=3$ or $d=6$, however, there are two possible quantizations and consequently two possibilities for the dimension of the dual operators. As we have discussed, however, for $d=6$ this ambiguity is removed by symmetry, which determines that the dual operators have dimension $\Delta_{+}=4$. But we are interested in the case $d=3$ here, which is the only case admitting non-supersymmetric fake superpotentials, and since there is an ambiguity in this case we will analyze the two possible quantizations separately. We will keep the analysis and the notation as general as possible, though, so that the analysis is applicable to other cases too.

Let us start by recalling that the asymptotic form of the potential (3.4) implies that a generic solution to the bulk scalar field equation of motion takes the form

$$
\phi(r, x) \sim e^{-\Delta_{-} r / l}\left(\phi_{-}(x)+\cdots\right)+e^{-\Delta_{+} r / l}\left(\phi_{+}(x)+\cdots\right) .
$$

Since we are excluding the case where the BF bound is saturated, we have $\Delta_{-}<\Delta_{+}$and so the term involving $\phi_{-}$dominates asymptotically as $r \rightarrow \infty$. For a particular solution,

\footnotetext{
${ }^{16}$ Curiously, this is precisely the range of dimensions for which there exist superconformal quantum field theories.
} 
however, such as a domain wall of the form (1.1), one of the functions $\phi_{ \pm}$can be zero. This depends entirely on the fake superpotential $W(\phi)$ that defines the flow equations (1.7). To determine the VEV of the operator dual to the scalar field $\phi$, one should evaluate the bulk action on the solution (6.6), which is identified with the generating functional of correlation functions of the dual operator [24].

Now, the on-shell action evaluated on a (Euclidean) Poincaré domain wall (1.1) is [2931

$$
S_{\text {on-shell }}^{B}=\int d^{d} x \sqrt{\gamma_{B}} W\left(\phi_{B}\right)
$$

where $\gamma_{B i j}=e^{2 A} \delta_{i j}$ and $W\left(\phi_{B}\right)$ is the fake superpotential that defines the flow equations (1.7). We have included the subscript $B$ here to emphasize that this is the on-shell action evaluated on the background domain wall solution. We will need to consider fluctuations around this background when we calculate two-point functions later on. As is well known, however, the on-shell action diverges and one needs to remove this divergence by adding covariant counterterms 32-34, 29, 35- 37, 12]. Although the covariant counterterms are a property of the supergravity action (1.2) - that is, once constructed in full generality by the asymptotic analysis of the action (1.2), they remove the divergences of the on-shell action when evaluated on all extrema of (1.2) - for domain wall backgrounds of the form (1.1) they take particularly simple form, which can be determined without the need to first compute the counterterms in full generality. In particular, the part of the the counterterm action that involves only the scalar fields, i.e. excluding the gravitational counterterms (except from the volume renormalization which can be counted with the scalar fields) and terms involving derivatives of the scalars (which vanish on the domain wall background), are given by a function $U(\phi)$ that satisfies equation (1.8) at least asymptotically [29, 37, 31], and has an expansion

$$
U(\phi)=-\frac{d-1}{\kappa^{2} l}-\frac{1}{2 l} \Delta_{-} \phi^{I} \phi^{I}+\mathcal{O}\left(\phi^{3}\right) .
$$

The first term in this function is nothing but the well-known volume renormalization term. The quadratic term requires some explanation, however.

Recall that, since $U(\phi)$ satisfies (1.8) and the potential has a Taylor expansion of the form (3.4), $U(\phi)$ has an expansion of the form (3.3) with the quadratic term being a diagonal matrix with diagonal elements $w_{ \pm}=-\Delta_{ \pm} / 2 l$ (see section 3i). ${ }^{17}$ There are $2^{n}$ such matrices, where $n$ is the number of independent scalars. But as we will now explain, there is a unique choice for the counterterms since they must remove the divergences for any fake superpotential $W(\phi)$, whose quadratic term can indeed be any of these $2^{n}$ matrices. It suffices to consider the case of a single on-shell scalar field, which takes the form (6.6). If $\phi_{-} \neq 0$, then its leading asymptotic behavior is $\phi \sim e^{-\Delta_{-} r / l} \phi_{-}$, and so, by the flow equations (1.7), the corresponding fake superpotential should have a quadratic term with coefficient $-\Delta_{-} / 2 l$. Since this is the same quadratic term as that of the counterterm $U(\phi)$, the quadratic term in the on-shell action will be canceled. In fact, one can take $U(\phi)$ to be the fake superpotential in this case - although this may not be necessary if there are

\footnotetext{
${ }^{17}$ Note that for $d>4$, however, $w_{ \pm}=-\Delta_{\mp} / 2 l$. Here we are primarily interested in the case $d=3$.
} 
no higher order divergences. It is crucial though that this same counterterm $U(\phi)$ removes the divergences for the case when $\phi_{-}$vanishes, since the counterterms are valid for any solution to a given bulk action. In this case $\phi \sim e^{-\Delta_{+} r / l} \phi_{+}$asymptotically and so the fake superpotential should have a quadratic term proportional to $-\Delta_{+} / 2 l$. This means that upon subtracting the counterterm $U(\phi)$, there will be a quadratic term $-\frac{1}{2 l}\left(\Delta_{+}-\Delta_{-}\right) \phi^{2}$ left in the action. However, $\phi^{2}=\mathcal{O}\left(e^{2 \Delta_{+} r / l}\right)$, in this case, and since $2 \Delta_{+}>d$, this term is not divergent and will drop out of the on-shell action as the regulator is removed. This argument explains why $\Delta_{-}$has to appear in the quadratic term of the counterterm. Generalizing this argument to more than one fields, ${ }^{18}$ the counterterm must have a quadratic term proportional to the unit matrix with coefficient $-\Delta_{-} / 2 l$.

Let us now apply this to the case we are interested in. From (6.5) we have

$$
\Delta_{-}=d-2, \quad d<4, \quad \Delta_{-}=2, \quad d>4 .
$$

Expanding the true superpotential (3.1) we get

$$
W_{o}(\phi)=-\frac{(d-1)}{\kappa_{D}^{2} l_{D}}-\frac{(d-2)}{2 l_{D}} \phi^{I} \phi^{I}+\frac{(d-2) \sqrt{2} \kappa_{D}}{96 l_{D}} \sum_{i=1}^{N} b_{i I} b_{i J} b_{i K} \phi^{I} \phi^{J} \phi^{K}+\mathcal{O}\left(\phi^{4}\right) .
$$

It follows that, for $d<4$, we can use $W_{o}(\phi)$ as the counterterm $U(\phi)$ :

$$
U(\phi)=W_{o}(\phi)
$$

A few comments are in order here. At first sight, it seems that for $d=6$ we are not able to use $W_{o}(\phi)$ as the counterterm since it has the wrong quadratic term, which is surprising since we know that $W_{o}(\phi)$ corresponds to a supersymmetric domain wall and, hence, one should be able to choose a supersymmetric renormalization scheme where the on-shell action is identically zero. The answer is that, as we showed in section 3, for $d=6$, the potential (3.4) requires that the quadratic term of any fake superpotential is $\operatorname{diag}\left(-\Delta_{+} / 2 l, \ldots,-\Delta_{+} / 2 l\right) .{ }^{19}$ Hence, there are simply no solutions with non-zero $\phi_{-}$in this case and so $W_{o}(\phi)$ can be safely used as the counterterm, resulting in the expected supersymmetric renormalization scheme. Second, focusing on the case $d=3$ which we are interested in, we have seen that there is a continuous family of fake superpotentials, of the generic form

$$
W(\phi)=-\frac{(d-1)}{\kappa_{D}^{2} l_{D}}-\frac{(d-2)}{2 l_{D}} \phi^{I} \phi^{I}+C_{I J K} \phi^{I} \phi^{J} \phi^{K}+\mathcal{O}\left(\phi^{4}\right)
$$

which have the same quadratic term as $W_{o}(\phi)$ and can therefore be used as the counterterm. They only differ from $W_{o}(\phi)$ at cubic order, which corresponds to a finite counterterm. In

\footnotetext{
${ }^{18}$ We assume that the scalar fields all have the same mass squared, but the argument generalizes in an obvious way to unequal masses.

${ }^{19}$ This fact, in combination with the fact that the dimension of the dual operators is unambiguously determined to be $\Delta_{+}=4$, implies that all domain walls for the $\operatorname{SL}(5, \mathbb{R}) / \mathrm{SO}(5)$ scalars in seven dimensions necessarily describe VEVs of the dual theory.
} 
principle, one is perfectly allowed to use any of these fake superpotentials as the counterterm, corresponding to a different renormalization scheme. However, since the counterterm is valid, and the same, for any solution of a given bulk action, there is a unique counterterm which ensures that the action vanishes for the supersymmetric domain wall solution defined by $W_{o}(\phi)$. Choosing any other counterterm would simply result in a non-supersymmetric renormalization scheme. Choosing the supersymmetric renormalization scheme, therefore, the renormalized on-shell action is given by

$$
S_{\mathrm{ren}}^{B}=\int d^{d} x \sqrt{\gamma_{B}}\left(W\left(\phi_{B}\right)-W_{o}\left(\phi_{B}\right)\right) .
$$

The analysis so far is independent of the dimension chosen for the dual operator. However, we will now see that, depending on such a choice, this renormalized action has different interpretations in the dual theory.

\section{$6.1 \Delta=\Delta_{+}$}

Consider first the more familiar case where the dimension, $\Delta$, of the dual operators $\mathcal{O}_{\Delta}^{I}$ is taken to be $\Delta_{+}$. The leading asymptotic term $\phi_{-}$in (6.6) corresponds then to the source of the dual operator, since $\Delta_{-}=d-\Delta_{+}$. In this case the generating functional of correlation functions is the renormalized on-shell action, which, evaluated on the background domain wall solution, takes the form (6.13). Using the Hamiltonian version of holographic renormalization, we find that the VEV of the dual stress tensor is related to the extrinsic curvature of the domain wall metric by 31]

$$
K_{j}^{i}=\dot{A} \delta_{j}^{i}=-\frac{\kappa^{2}}{d-1} W(\phi) \delta_{j}^{i} .
$$

In particular, the renormalized expectation value of the stress tensor is given by

$$
\left\langle T_{j}^{i}\right\rangle_{\mathrm{ren} .}=-\frac{1}{\kappa^{2}}\left(K_{(d) j}^{i}-K_{(d)} \delta_{j}^{i}\right)=-(W(\phi)-U(\phi)) \delta_{j}^{i} .
$$

Moreover, the renormalized VEV of the scalar operators is

$$
\left\langle\mathcal{O}_{\Delta_{+}}^{I}\right\rangle_{\text {ren. }}=\frac{\partial}{\partial \phi^{I}}(W(\phi)-U(\phi)) .
$$

The value of these one-point functions depends on the form of $W(\phi)$, and in particular, on the quadratic one, but possibly on higher order terms as well. To be concrete, let us return to the case $d=3$ and the $\mathrm{SL}(8, \mathbb{R}) / \mathrm{SO}(8)$ scalars. Recall that in this case the most general fake superpotential $W(\phi)$ has two possible forms. First, there is a continuous family of fake superpotentials whose quadratic term is the same as that of $W_{o}(\phi)$, but have arbitrary cubic term. The Taylor expansion of these fake superpotentials takes the form (6.12). Evaluating the one-point functions in this case gives

$$
\left\langle T_{j}^{i}\right\rangle_{+}=-\left(C_{I J K}-C_{o I J K}\right) \phi_{B}^{I} \phi_{B}^{J} \phi_{B}^{K} \delta_{j}^{i}, \quad\left\langle\mathcal{O}_{\Delta_{+}}^{I}\right\rangle=3\left(C_{I J K}-C_{o I J K}\right) \phi_{B}^{J} \phi_{B}^{K},
$$

where $C_{O I J K}=\frac{\sqrt{2} \kappa}{96 l} \sum_{i=1}^{8} b_{i I} b_{i J} b_{i K}$ is the cubic coefficient of $W_{o}(\phi)$ and the subscript + is a reminder that the VEV is taken in the theory where the operators dual to the scalar fields 
have dimension $\Delta_{+}$. It is understood that these are the renormalized VEVs. There is also a second class of fake superpotentials which have vanishing cubic term, but whose quadratic term can be different from that of $W_{o}(\phi)$. Namely, the diagonal elements of the matrix multiplying the quadratic term of $W(\phi)$ can be either $-\Delta_{+} / 2 l$ or $-\Delta_{-} / 2 l$. Depending on which of these two values the $I$-th component takes, in this case the one-point functions are given by

$$
\left\langle T_{j}^{i}\right\rangle_{+}=C_{o I J K} \phi_{B}^{I} \phi_{B}^{J} \phi_{B}^{K} \delta_{j}^{i}, \quad\left\langle\mathcal{O}_{\Delta_{+}}^{I}\right\rangle= \begin{cases}-3 C_{o I J K} \phi_{B}^{J} \phi_{B}^{K}, & -\Delta_{-} / 2 l, \\ -3 C_{o I J K} \phi_{B}^{J} \phi_{B}^{K}+\frac{1}{l}\left(d-2 \Delta_{+}\right) \phi_{B}^{I}, & -\Delta_{+} / 2 l .\end{cases}
$$

Note that the quadratic term of $W(\phi)$ does not contribute to the VEV of the stress tensor since $2 \Delta_{+}>d$. Moreover, in the terms involving $C_{O I J K}$ only the scalars for which the diagonal matrix multiplying the quadratic term of $W(\phi)$ has values $-\Delta_{-} / 2 l$ contribute, since $3 \Delta_{-}=d$ and $2 \Delta_{-}=\Delta_{+}$. The components involving scalars with a quadratic term $-\Delta_{+} / 2 l$ in the fake superpotential do not contribute to these terms. We should emphasize that although we do not in general know the full fake superpotentials, the onepoint functions we have calculated are in fact exact, since they only depend on the quadratic and cubic terms of the fake superpotential. As a check, one can easily verify that in both cases, the Ward identity

$$
\left\langle T_{i}^{i}\right\rangle_{+}=-\sum_{I}\left(d-\Delta_{+}\right) \phi_{B}^{I}\left\langle\mathcal{O}_{\Delta_{+}}^{I}\right\rangle
$$

is satisfied. Finally, for future reference, let us give more explicitly the VEVs for the one-scalar solutions of section 4.1. Namely,

$$
\begin{array}{lll}
\left\langle T_{j}^{i}\right\rangle_{+}=\frac{\kappa}{l} \frac{16\left(\alpha-\alpha_{o}\right)}{(2 k(8-k))^{3 / 2}} \phi_{B}^{3} \delta_{j}^{i}, & \left\langle\mathcal{O}_{\Delta_{+}}\right\rangle=-\frac{3 \kappa}{l} \frac{16\left(\alpha-\alpha_{o}\right)}{(2 k(8-k))^{3 / 2}} \phi_{B}^{2}, & \text { for } W(\phi ; \alpha), \\
\left\langle T_{j}^{i}\right\rangle_{+}=0, & \left\langle\mathcal{O}_{\Delta_{+}}\right\rangle=-\frac{1}{l} \phi_{B}, & \text { for } \widetilde{W}_{o}(\phi),
\end{array}
$$

and recall that $\alpha_{o}=-(8-k)(k-4) k / 24$.

The VEVs we have just computed show that if one associates the $\mathrm{SL}(8, \mathbb{R}) / \mathrm{SO}(8)$ scalars with operators of dimension $\Delta_{+}=2$, the supersymmetric domain walls corresponding to $W_{o}(\phi)$ have zero VEVs and hence describe a deformation of the CFT Lagrangian. The non-supersymmetric domain walls corresponding to $\widetilde{W}_{o}(\phi)$, however, describe a nonconformal and non-supersymmetric vacuum. Moreover, the continuous family of domain walls defined by $W(\phi ; \alpha)$, with $\alpha \neq \alpha_{o}$, gives VEVs to both the stress tensor and the scalar operators, but these are non-linear in the scalar source. These VEVs are on top of the deformation corresponding to $W_{o}(\phi)$ and they break supersymmetry.

$6.2 \Delta=\Delta_{-}$

Consider the case where the dimension $\Delta$ of the dual operator is $\Delta_{-}$. Of course, the leading asymptotic behavior of the scalar field (6.6) is still the term involving $\phi_{-}$, but now it cannot be identified with the source of the dual operator since it has the wrong 
asymptotic behavior for being the source. This means that the renormalized action (6.13) cannot be the generating functional of correlation functions of the operator $\mathcal{O}_{\Delta_{-}}$. So a more careful analysis is required in this case.

As we have already pointed out, the evaluation of the renormalized action by adding covariant counterterms is not affected by the question of whether the dual operator has dimension $\Delta_{+}$or $\Delta_{-}$. The only difference arises in the identification of the functional that generates the corresponding correlation functions of the dual operator. In any case, therefore, following the standard procedure, we need to evaluate the renormalized on-shell action, which we now call $I\left[\phi_{-}\right]$. This is a functional of $\phi_{-}-$independently of which choice for the dimension of the dual operator is made - since the supergravity equations of motion with Dirichlet boundary conditions express $\phi_{+}$as a functional of $\phi_{-}$. If the dimension of the dual operator is $\Delta_{+}$, as we have seen $\phi_{-}$corresponds to the source of the operator and, hence, $I\left[\phi_{-}\right]$can be identified with the generating functional of connected correlators of $\mathcal{O}_{\Delta_{+}}$. If the dimension of the dual operator is $\Delta_{-}$, however, this identification cannot be made since, the still arbitrary function $\phi_{-}$, does not correspond to the source of $\mathcal{O}_{\Delta_{-}}$. In [24] it was suggested that in this case the correct generating functional is obtained from $I\left[\phi_{-}\right]$by a Legendre transformation as

$$
L\left[\bar{\phi}_{-}, \phi_{-}\right]=I\left[\phi_{-}\right]+\int d^{d} x \sqrt{g_{(0)}} \bar{\phi}_{-}(x) \phi_{-}(x),
$$

where $g_{(0) i j}$ is the boundary metric. Extremizing $L\left[\bar{\phi}_{-}, \phi_{-}\right]$with respect to $\phi_{-}$, gives

$$
\bar{I}\left[\bar{\phi}_{-}\right] \equiv L\left[\bar{\phi}_{-}, \phi_{-}^{*}\left(\bar{\phi}_{-}\right)\right]
$$

where $\phi_{-}^{*}\left(\bar{\phi}_{-}\right)$is the solution to

$$
\left.\frac{\delta L\left[\bar{\phi}_{-}, \phi_{-}\right]}{\delta \phi_{-}}\right|_{\phi_{-}^{*}}=\left.\frac{\delta I\left[\phi_{-}\right]}{\delta \phi_{-}}\right|_{\phi_{-}^{*}}+\bar{\phi}_{-}(x)=\left\langle\mathcal{O}_{\Delta_{+}}\right\rangle_{\phi_{-}=\phi_{-}^{*}}+\bar{\phi}_{-}(x)=0 .
$$

$\bar{I}\left[\bar{\phi}_{-}\right]$is now identified with the generating functional of connected correlation functions of the operator $\mathcal{O}_{\Delta_{-}}$and $\bar{\phi}_{-}(x)$ is identified with the source of $\mathcal{O}_{\Delta_{-}}{ }^{20}$ In particular, the exact and renormalized one-point function of $\mathcal{O}_{\Delta_{-}}$in the presence of a source is

$$
\left\langle\mathcal{O}_{\Delta_{-}}\right\rangle_{\bar{\phi}_{-}} \equiv \frac{\delta \bar{I}\left[\bar{\phi}_{-}\right]}{\delta \bar{\phi}_{-}}=\phi_{-}^{*}\left(\bar{\phi}_{-}\right)
$$

which is simply the solution to 6.23). The last two equations tell us that the one-point function of the operator $\mathcal{O}_{\Delta_{-}}$is proportional to the source, $\phi_{-}$, of the operator $\mathcal{O}_{\Delta_{+}}$and vice versa. We can now check that an analogue of the Ward identity (6.19) holds for this case too. First we note that the stress tensors corresponding to the two generating functionals $I$ and $\bar{I}$ are related by

$$
\left\langle T_{i j}\right\rangle_{\bar{\phi}_{-}} \equiv \frac{2}{\sqrt{g_{(0)}}} \frac{\delta \bar{I}\left[\bar{\phi}_{-}, g_{(0)}\right]}{\delta g_{(0)}{ }^{i j}}=\left\langle T_{i j}\right\rangle_{\phi_{-}}-g_{(0) i j} \bar{\phi}_{-I} \phi_{-}^{I} .
$$

\footnotetext{
${ }^{20}$ Note that $\bar{\phi}_{-}(x) \sim \phi_{+}(x)$ up to some numerical factor. See e.g. 38].
} 
Using now the Ward identity (6.19) for $\left\langle T_{i}^{i}\right\rangle_{\phi_{-}}$together with the relations $\left\langle\mathcal{O}_{\Delta_{+}}^{I}\right\rangle=-\bar{\phi}_{-I}$ and $\left\langle\mathcal{O}_{\Delta_{-}}^{I}\right\rangle=\phi_{-}^{I}$, we obtain

$$
\left\langle T_{i}^{i}\right\rangle_{\bar{\phi}_{-}}=-\sum_{I}\left(d-\Delta_{-}\right) \bar{\phi}_{-I}\left\langle\mathcal{O}_{\Delta_{-}}^{I}\right\rangle
$$

where we have used $\Delta_{-}+\Delta_{+}=d$.

We can now evaluate the one-point function of the operators $\mathcal{O}_{\Delta_{-}}^{I}$ in the background domain wall solutions very easily. Starting from the renormalized on-shell action (6.13), we can immediately evaluate the one-point functions by solving (6.23), which in this case reads:

$$
\frac{\partial}{\partial \phi^{I}}\left(W(\phi)-W_{o}(\phi)\right)+\bar{\phi}_{-I}=0 .
$$

Comparing this to (6.16), we see that the source $\bar{\phi}_{-}$of the operator $\mathcal{O}_{\Delta_{-}}$is proportional to the VEV of the operator $\mathcal{O}_{\Delta_{+}}$. It follows that asymptotically $\bar{\phi}_{-}=\mathcal{O}\left(e^{-\Delta_{+} r / l}\right)=$ $\mathcal{O}\left(e^{-\left(d-\Delta_{-}\right) r / l}\right)$, as is required for the source of an operator of dimension $\Delta_{-}$. This equation can be used to determine $\phi_{B}\left(\bar{\phi}_{-B}\right)$, but since the domain wall solution is given in terms of $\phi_{B}^{I}$ and not $\bar{\phi}_{-B}^{I}$, we can evaluate the VEVs in terms of $\phi_{B}^{I}$, instead of $\bar{\phi}_{-B}^{I}$. From (6.24) we get, depending on the coefficient of the quadratic term in $W(\phi)$,

$$
\left\langle\mathcal{O}_{\Delta_{-}}^{I}\right\rangle=\left\{\begin{array}{c}
\phi_{B}^{I},-\Delta_{-} / 2 l, \\
0,-\Delta_{+} / 2 l .
\end{array}\right.
$$

In this case, therefore, the VEVs are much simpler and completely independent of the cubic term in $W(\phi)$. Moreover, from $(\underline{6.25})$ we find

$$
\left\langle T_{j}^{i}\right\rangle_{-}=2\left(C_{I J K}-C_{o I J K}\right) \phi_{B}^{I} \phi_{B}^{J} \phi_{B}^{K} \delta_{j}^{i},
$$

for the continuous family of fake superpotentials which has a coefficient $-\Delta_{-} / 2 l$ for the quadratic term of all scalar fields, while

$$
\left\langle T_{j}^{i}\right\rangle_{-}=-2 C_{o I J K} \phi_{B}^{I} \phi_{B}^{J} \phi_{B}^{K} \delta_{j}^{i},
$$

for the superpotentials that have a vanishing cubic term but any combination of $-\Delta_{ \pm} / 2 l$ for the quadratic term. Again, only the scalar fields with $-\Delta_{-} / 2 l$ in the quadratic term contribute to the last expression.

We see that the role of $W_{o}(\phi)$ and $\widetilde{W}_{o}(\phi)$ have been interchanged now compared to the case where the dual operators have dimension $\Delta_{+}$. Namely, the supersymmetric domain walls now describe a non-conformal but supersymmetric vacuum, which has been identified with the Coulomb branch of the dual CFT [16], while the non-supersymmetric domain walls corresponding to $\widetilde{W}_{o}(\phi)$ describe a (single-trace) deformation of the CFT Lagrangian. The domain walls described by the continuous family of fake superpotentials $W(\phi ; \alpha)$ correspond to a line of marginal triple-trace deformations of the Coulomb branch. We stress that this does not mean that the theory has a flat direction. At the supersymmetric point, i.e. the Coulomb branch, the scalar operator can have an arbitrary VEV. If the marginal triple-trace deformation is turned on, it produces a potential for the VEV 
of the scalar operator forcing it to zero. Nevertheless, it is possible to give an arbitrary VEV to this operator in this case too, provided we simultaneously turn on a source for the single-trace operator, proportional to the marginal triple-trace deformation parameter. This single-trace deformation breaks conformal invariance explicitly, which justifies the fact that the trace of the stress tensor in (6.29) is non-zero. Spontaneous breaking of the conformal symmetry only occurs at the supersymmetric point corresponding to no deformation. These combined marginal triple-trace and induced single-trace deformations allowing for an arbitrary VEV is precisely what is described by the non-supersymmetric domain walls corresponding to $W(\phi ; \alpha)$.

\section{Holographic two-point functions}

To further understand the RG flows described by the domain walls we have discussed, we now turn to the computation of the holographic two-point functions. However, even for the supersymmetric domain walls with a single scalar field turned on, the linearized bulk equations of motion that we need to solve cannot always be solved analytically. We will therefore focus on a single scalar field, and in particular on the case $k=4$ for which the twopoint functions corresponding to the supersymmetric background can be computed exactly. Unfortunately for $k=4$ we do not have the full non-perturbative fake superpotentials $W(\phi ; \alpha)$ or $\widetilde{W}_{o}(\phi)$ as for $k=6$, but we do have $W(\phi ; \alpha)$ perturbatively in $\alpha-\alpha_{o}$ and we can therefore compute the two-point functions for the corresponding non-supersymmetric backgrounds perturbatively in the parameter $\alpha-\alpha_{o}$. Of course, this will provide us with no information on the domain wall defined by $\widetilde{W}_{o}(\phi)$, however. To evaluate these two-point functions we will follow the approach suggested in [12], where the relevant counterterms can be evaluated directly from the linearized equations, without the need for the computation of the full non-linear set of counterterms required in general for the gravity-scalar action. Indeed, as we will see, the counterterms that we will need are almost trivial. For earlier work on the holographic computation of correlation functions see [39-41, 30, 42].

To calculate the sought after two-point functions, we need to linearize the bulk equations of motion around the domain wall background (1.1). To this end, we write the bulk metric in the form

$$
d s^{2}=d r^{2}+\gamma_{i j}(r, x) d x^{i} d x^{j},
$$

and consider linear fluctuations

$$
\gamma_{i j}=\gamma_{B i j}(r)+h_{i j}(r, x)=e^{2 A(r)} \delta_{i j}+h_{i j}(r, x), \quad \phi=\phi_{B}(r)+\varphi(r, x) .
$$

The extrinsic curvature, $K_{i j}=\frac{1}{2} \dot{\gamma}_{i j}$, then becomes

$$
K_{j}^{i}=\dot{A} \delta_{j}^{i}+\frac{1}{2} \dot{S}_{j}^{i}
$$

where $S_{j}^{i} \equiv \gamma_{B}^{i k} h_{k j}$. Next we decompose $S_{j}^{i}$ into irreducible components as

$$
S_{j}^{i}=e_{j}^{i}+\partial^{i} \epsilon_{j}+\partial_{j} \epsilon^{i}+\frac{d}{d-1}\left(\frac{1}{d} \delta_{j}^{i}-\frac{\partial^{i} \partial_{j}}{\square_{B}}\right) f+\frac{\partial^{i} \partial_{j}}{\square_{B}} S
$$


where $\partial_{i} e_{j}^{i}=e_{i}^{i}=\partial_{i} \epsilon^{i}=0, \square_{B}=e^{-2 A} \square=e^{-2 A} \delta^{i j} \partial_{i} \partial_{j}$, and indices are raised with the inverse background metric $e^{-2 A} \delta^{i j}$. Conversely, the projection operators

$$
\Pi_{k j}^{i l}=\frac{1}{2}\left(\pi_{k}^{i} \pi_{j}^{l}+\pi^{i l} \pi_{k j}-\frac{2}{d-1} \pi_{j}^{i} \pi_{k}^{l}\right),
$$

and

$$
\pi_{j}^{i}=\delta_{j}^{i}-\frac{\partial^{i} \partial_{j}}{\square_{B}}
$$

allow one to uniquely express each of the irreducible components in terms of $S_{j}^{i}$ as

$$
e_{j}^{i}=\Pi_{k}^{i}{ }_{k}^{l} S_{l}^{k}, \quad \epsilon_{i}=\pi_{i}^{l} \frac{\partial_{k}}{\square_{B}} S_{l}^{k}, \quad f=\pi_{k}^{l} S_{l}^{k}, \quad S=\delta_{k}^{l} S_{l}^{k} .
$$

The linearized equations for these modes are [12]

$$
\begin{gathered}
\left(\partial_{r}^{2}+d \dot{A} \partial_{r}+e^{-2 A} \square\right) e_{j}^{i}=0, \\
\left(\partial_{r}^{2}+\left[d \dot{A}+2 W \partial_{\phi}^{2} \log W\right] \partial_{r}+e^{-2 A} \square\right) \omega=0, \\
\dot{f}=-2 \kappa^{2} \dot{\phi}_{B} \varphi, \\
\dot{S}=\frac{1}{(d-1) \dot{A}}\left[-e^{-2 A} \square f+2 \kappa^{2}\left(\dot{\phi}_{B} \dot{\varphi}-V^{\prime}\left(\phi_{B}\right) \varphi\right)\right],
\end{gathered}
$$

where

$$
\omega \equiv \frac{W}{W^{\prime}} \varphi+\frac{1}{2 \kappa^{2}} f
$$

Note that in writing the linearized equations in this form we have used the diffeomorphism invariance in the transverse space to set $\epsilon_{i} \equiv 0$. The exact, unrenormalized, one-point functions in the presence of linear sources are given by the canonical momenta $\dot{e}_{j}^{i}, \dot{\omega}$ etc. The last two equations give immediately the momenta dual to $f$ and $S$. To determine the momenta for $e_{j}^{i}$ and $\omega$ we note that, to linear order, we must have 12]

$$
\dot{e}_{j}^{i}=E\left(A, \phi_{B}\right) e_{j}^{i}, \quad \dot{\omega}=\Omega\left(A, \phi_{B}\right) \omega .
$$

Inserting these relations into the first two equations in (7.8), we obtain two first order equations for $E$ and $\Omega$

$$
\begin{aligned}
\dot{E}+E^{2}+d \dot{A} E-e^{-2 A} p^{2} & =0, \\
\dot{\Omega}+\Omega^{2}+\left[d \dot{A}+2 W \partial_{\phi}^{2} \log W\right] \Omega-e^{-2 A} p^{2} & =0,
\end{aligned}
$$

where $p$ denotes the transverse space momentum. All canonical momenta can now be easily expressed in terms of $E$ and $\Omega$. From (7.8) we deduce

$$
\begin{aligned}
\dot{e}_{j}^{i} & =E e_{j}^{i}, \\
\dot{f} & =-2 \kappa^{2} W^{\prime} \varphi \\
\dot{\varphi} & =\left(W^{\prime \prime}+\Omega\right) \varphi+\frac{1}{2 \kappa^{2}} \frac{W^{\prime}}{W} \Omega f \\
\dot{S} & =-\frac{1}{\kappa^{2}}\left[\left(\frac{W^{\prime}}{W}\right)^{2} \Omega+\frac{e^{-2 A}}{W} p^{2}\right] f-2 \frac{W^{\prime}}{W}\left(\Omega+\frac{d \kappa^{2}}{d-1} W\right) \varphi .
\end{aligned}
$$


As we have pointed out, these are the unrenormalized momenta. To get the renormalized momenta we need to add appropriate counterterms. In the Hamiltonian formulation of holographic renormalization the counterterms for the canonical momenta are simply computed by expanding the latter in eigenfunctions of the dilatation operator [12]

$$
\delta_{D} \equiv \int d^{d} x 2 \gamma_{i j} \frac{\delta}{\delta \gamma_{i j}}+(\Delta-d) \int d^{d} x \phi \frac{\delta}{\delta \phi}
$$

As long as we are interested in the canonical momenta to linear order in the sources, which is sufficient for computing the two-point functions, expanding the expressions (7.12) in eigenfunctions of the dilatation operator is particularly simple, since we only need to expand the coefficients of the linear fluctuations. These are only functions of the background fields $A$ and $\phi_{B}$ and so the dilatation operator simplifies to

$$
\delta_{D}=\partial_{A}+(\Delta-d) \phi_{B} \partial_{\phi_{B}}
$$

It is important to keep in mind that, although the covariant counterterms for the canonical momenta are computed directly by expanding the canonical momenta in eigenfunctions of the dilatation operator, the same result would be obtained by first computing the covariant counterterms for the on-shell action and then deriving the renormalized momenta from the renormalized on-shell action. Indeed, the ability to compute the renormalized momenta, which are linear in the fluctuations, without first having to compute the renormalized on-shell action, which is quadratic in the fluctuations, is one of the advantages of the Hamiltonian approach. One must, however, ensure that the counterterms for the canonical momenta correspond to a given renormalization scheme, which is usually determined by fixing the value of the renormalized on-shell action on a given background. Since the only non-vanishing contribution to the counterterms, when evaluated on the background domain wall, is the function $U(\phi)$ (see section (6), a given renormalization scheme is defined by a choice of $U(\phi)$. It follows that the renormalized momenta will automatically be compatible with the chosen renormalization scheme once the contribution of $U(\phi)$ to the counterterms of the canonical momenta has been taken into account. As we have seen in section 6, for the domain walls we are interested in here we can take $U(\phi)$ to be the superpotential $W_{o}(\phi)$, corresponding to a supersymmetric renormalization scheme. The corresponding counterterm

$$
-\int d^{d} x \sqrt{\gamma} W_{o}\left(\phi_{B}+\varphi\right)
$$

leads to the following contributions to the canonical momenta:

$$
\begin{aligned}
& \dot{S}_{j}^{i}: \frac{2 \kappa^{2}}{(d-1)} W_{o}^{\prime}\left(\phi_{B}\right) \varphi \delta_{j}^{i} \\
& \dot{\varphi}:-W_{o}^{\prime \prime}\left(\phi_{B}\right) \varphi .
\end{aligned}
$$


Adding these contributions to the canonical momenta (7.12) we obtain

$$
\begin{aligned}
\dot{e}_{j}^{i} & =E e_{j}^{i}, \\
\dot{f} & =-2 \kappa^{2}\left(W^{\prime}-W_{o}^{\prime}\right) \varphi \\
\dot{\varphi} & =\left(W^{\prime \prime}-W_{o}^{\prime \prime}+\Omega\right) \varphi+\frac{1}{2 \kappa^{2}} \frac{W^{\prime}}{W} \Omega f, \\
\dot{S} & =-\frac{1}{\kappa^{2}}\left[\left(\frac{W^{\prime}}{W}\right)^{2} \Omega+\frac{e^{-2 A}}{W} p^{2}\right] f-2\left(\frac{W^{\prime}}{W} \Omega+\frac{d \kappa^{2}}{d-1}\left(W^{\prime}-W_{o}^{\prime}\right)\right) \varphi .
\end{aligned}
$$

These are not yet the renormalized momenta, but it is now guaranteed that by expanding these canonical momenta in eigenfunctions of the dilatation operator and keeping the terms of weight $d=3$ for $\dot{e}_{j}^{i}, \dot{f}$ and $\dot{S}$, and of weight $\Delta_{+}=2$ for $\dot{\varphi}$, we obtain the renormalized momenta that correspond to the supersymmetric scheme defined by $W_{o}$.

Since $W\left(\phi_{B}\right)$ and $W_{o}\left(\phi_{B}\right)$ are known functions of the background fields, the only nontrivial step in computing the renormalized canonical momenta is determining the expansion of $E$ and $\Omega$ in eigenfunctions of the dilatation operator. This can be easily done by using the equations (7.11). One expands the radial derivative as

$$
\partial_{r}=\dot{A} \partial_{A}+\dot{\phi}_{B} \partial_{\phi_{B}}=-\frac{\kappa^{2}}{d-1} W\left(\phi_{B}\right) \partial_{A}+W^{\prime}\left(\phi_{B}\right) \partial_{\phi_{B}} \sim \delta_{D}+\cdots,
$$

as well as the functions $E$ and $\Omega^{21}$

$$
\begin{aligned}
& E=E_{(1)}+\cdots+E_{(d)}+\cdots, \\
& \Omega=\Omega_{(0)}+\cdots+\Omega_{(2 \Delta-d)}+\cdots,
\end{aligned}
$$

and inserts these expansions in equations (7.11). Collecting terms of the same dilatation weight then determines all terms in the expansions (7.19), except for the coefficients $E_{(d)}$ and $\Omega_{(2 \Delta-d)}$. These terms contain all dynamical information about the two-point functions and can only be determined by solving exactly the first two equations in (7.8) or, equivalently, equations (7.11). There is, however, an important technical difference between solving the former or the latter. By solving the linear second order equations in (7.8), one obtains two linearly independent solutions, namely the 'normalizable' and 'non-normalizable' modes. Generically, an arbitrary linear combination of these solutions will have a singularity somewhere in the interior of the asymptotically AdS space. However, there is usually a unique linear combination which leads to a non-singular solution. This requirement determines the coefficient of the normalizable mode in terms of the nonnormalizable mode, which should be arbitrary since it corresponds to the source of the dual operator. If one instead solves the first order equations (7.11) only one integration constant appears instead of two, which simply reflects the fact that the overall normalization of the linearized solutions of (7.8) has been factored out from $E$ and $\Omega$. The integration constant in $E$ and $\Omega$ can therefore be understood as the ratio of the normalizable and

\footnotetext{
${ }^{21}$ In general one would have to include logarithmic terms in these expansions, but in our case we do not need them since the boundary is three-dimensional. See 12 for the general case.
} 
non-normalizable modes of the solutions to the second order equations (7.8). However, it is not always possible to determine this integration constant by the requirement that the exact solutions for $E$ and $\Omega$ are non-singular. This is because it is possible that $E$ and $\Omega$ are non-singular, even though the corresponding solutions of the second order equations (7.8) are singular. Practically, therefore, to obtain the correct exact solution for $E$ and $\Omega$, one should first solve the corresponding second order equations (7.8), demand that they are non-singular, and then deduce the corresponding $E$ and $\Omega$. Equations (7.11) are still essential, however, for determining the covariant counterterms for $E$ and $\Omega$.

We compute $E_{(3)}$ and $\Omega_{(1)}$ explicitly in appendix $\square$ for the $k=4$ one-scalar domain wall. The result is given in C.7. Given these quantities one can now determine the onepoint functions with linearized sources and, consequently, the exact two-point functions. Expanding the canonical momenta (7.17) one easily deduces that the renormalized onepoint functions are given by

$$
\begin{aligned}
\left\langle T_{j}^{i}\right\rangle_{+} & =\frac{\kappa \alpha}{8 \sqrt{2} l} \phi_{B}^{3} \delta_{j}^{i}-\frac{1}{2 \kappa^{2}} E_{(3)} e_{(0)}^{i}-\frac{1}{8} \phi_{B}^{2} \pi_{j}^{i} \Omega_{(1)} f_{(0)}+\left(\frac{3 \kappa \alpha}{8 \sqrt{2} l} \phi_{B}^{2} \delta_{j}^{i}-\frac{1}{2} \phi_{B} \pi_{j}^{i} \Omega_{(1)}\right) \varphi_{(0)}, \\
\left\langle\mathcal{O}_{\Delta_{+}}\right\rangle & =-\frac{3 \kappa \alpha}{8 \sqrt{2} l} \phi_{B}^{2}+\frac{1}{4} \phi_{B} \Omega_{(1)} f_{(0)}+\left(-\frac{3 \kappa \alpha}{4 \sqrt{2} l} \phi_{B}+\Omega_{(1)}\right) \varphi_{(0)} .
\end{aligned}
$$

Again, one should keep in mind that these are the one-point functions when the dual scalar operators are taken to have dimension $\Delta_{+}$. We will consider the case $\Delta_{-}$below. It is reassuring that these one-point functions satisfy the Ward identity (6.19) as they should. Differentiating with respect to the linear sources one finally obtains the two-point functions

$$
\begin{aligned}
\left\langle T_{j}^{i} T_{l}^{k}\right\rangle & =-\frac{1}{\kappa^{2}} \Pi^{i}{ }_{l}^{k}{ }_{j} E_{(3)}-\frac{1}{4} \phi_{B}^{2} \pi_{j}^{i} \pi_{l}^{k} \Omega_{(1)}, \\
\left\langle T_{j}^{i} \mathcal{O}_{\Delta_{+}}\right\rangle & =-\frac{3 \kappa \alpha}{8 \sqrt{2} l} \phi_{B}^{2} \delta_{j}^{i}+\frac{1}{2} \phi_{B} \pi_{j}^{i} \Omega_{(1)}, \\
\left\langle\mathcal{O}_{\Delta_{+}} \mathcal{O}_{\Delta_{+}}\right\rangle & =\frac{3 \kappa \alpha}{4 \sqrt{2} l} \phi_{B}-\Omega_{(1)} .
\end{aligned}
$$

The two-point functions for the case where the dual scalar operators have dimension $\Delta_{-}$can also be deduced from the one-point functions (7.20). As we have seen in the previous section, the source dual to $\mathcal{O}_{\Delta_{-}}$is given by the VEV of $\mathcal{O}_{\Delta_{+}}$as

$$
\bar{\phi}=\bar{\phi}_{B}+\bar{\varphi}=-\left\langle\mathcal{O}_{\Delta_{+}}\right\rangle
$$

from which we infer

$$
\begin{aligned}
\varphi & =\left(\frac{3 \kappa \alpha}{4 \sqrt{2} l} \phi_{B}-\Omega_{(1)}\right)^{-1}\left(\bar{\varphi}+\frac{1}{4} \phi_{B} \Omega_{(1)} f_{(0)}\right) \\
& =-\Omega_{(1)}{ }^{-1}\left(1+\frac{3 \kappa \alpha}{4 \sqrt{2} l} \phi_{B} \Omega_{(1)}^{-1}+\mathcal{O}\left(\alpha^{2}\right)\right)\left(\bar{\varphi}+\frac{1}{4} \phi_{B} \Omega_{(1)} f_{(0)}\right) .
\end{aligned}
$$

It follows that

$$
\left\langle\mathcal{O}_{\Delta_{-}}\right\rangle=\left(\phi_{B}+\varphi\right)=\phi_{B}-\left(1+\frac{3 \kappa \alpha}{4 \sqrt{2} l} \phi_{B} \Omega_{(1)}^{-1}+\mathcal{O}\left(\alpha^{2}\right)\right)\left(\Omega_{(1)}^{-1} \bar{\varphi}+\frac{1}{4} \phi_{B} f_{(0)}\right) .
$$


Moreover, from (6.25) we obtain

$$
\begin{aligned}
\left\langle T_{j}^{i}\right\rangle_{-}= & \left\langle T_{j}^{i}\right\rangle_{+}-\bar{\phi}\left\langle\mathcal{O}_{\Delta_{-}}\right\rangle \delta_{j}^{i} \\
= & -\frac{\kappa \alpha}{4 \sqrt{2} l} \phi_{B}^{3} \delta_{j}^{i}-\frac{1}{2 \kappa^{2}} E_{(3)} e_{(0) j}{ }_{j}^{i}-\phi_{B}\left(\delta_{j}^{i}-\frac{1}{2} \pi_{j}^{i}\left(1+\frac{3 \kappa \alpha}{4 \sqrt{2} l} \phi_{B} \Omega_{(1)}{ }^{-1}+\mathcal{O}\left(\alpha^{2}\right)\right)\right) \bar{\varphi} \\
& +\frac{3 \kappa \alpha}{32 \sqrt{2} l} \phi_{B}^{3} \pi_{j}^{i} f_{(0)} .
\end{aligned}
$$

Once again, these one-point functions satisfy the Ward identity (6.26) as required. Differentiating with respect to the sources we now obtain the two-point functions

$$
\begin{aligned}
\left\langle T_{j}^{i} T_{l}^{k}\right\rangle & =-\frac{1}{\kappa^{2}} \Pi^{i}{ }_{l}{ }_{j} E_{(3)}+\frac{3 \kappa \alpha}{16 \sqrt{2} l} \phi_{B}^{3} \pi_{j}^{i} \pi_{l}^{k}, \\
\left\langle T_{j}^{i} \mathcal{O}_{\Delta_{-}}\right\rangle & =\phi_{B}\left(\delta_{j}^{i}-\frac{1}{2} \pi_{j}^{i}\left(1+\frac{3 \kappa \alpha}{4 \sqrt{2} l} \phi_{B} \Omega_{(1)}{ }^{-1}+\mathcal{O}\left(\alpha^{2}\right)\right)\right), \\
\left\langle\mathcal{O}_{\Delta_{-}} \mathcal{O}_{\Delta_{-}}\right\rangle & =\Omega_{(1)}{ }^{-1}\left(1+\frac{3 \kappa \alpha}{4 \sqrt{2} l} \phi_{B} \Omega_{(1)}{ }^{-1}+\mathcal{O}\left(\alpha^{2}\right)\right) .
\end{aligned}
$$

In order to discuss the physics of these two-point functions, it is useful to reinstate the dependence on the $M 2$-brane distribution parameters, which we have so far suppressed because this is different for different domain walls. For the case $k=4$ for which we have computed the two-point functions, the $M 2$-branes are distributed on an $S^{3}$ of radius $l_{1}$. By uplifting our domain wall solution to eleven-dimensions we find that the scalar VEV is as expected proportional to the radius of the $M 2$-branes distribution, $\phi_{B}=\sqrt{2} l_{1} / \kappa l$, while the momenta, $\tilde{p}^{i}$, on the world-volume of the $M 2$-branes are related to the momenta above by $p^{i}=\left(l / l_{1}\right) \tilde{p}^{i}$. With these relations and the result (C.7) for $\Omega_{(1)}$ we can write the scalar two-point function as

$$
\left\langle\mathcal{O}_{\Delta_{-}} \mathcal{O}_{\Delta_{-}}\right\rangle=\frac{2}{\tilde{p}^{2}} \sqrt{\tilde{p}^{2}+\frac{4 l_{1}^{2}}{l^{4}}}\left(1-\alpha l_{1} \frac{\left(9 l^{8} \tilde{p}^{4}+32 l_{1}^{2} l^{4} \tilde{p}^{2}+16 l_{1}^{4}\right)}{2 l^{10} \tilde{p}^{2}\left(\tilde{p}^{2}+4 l_{1}^{2} / l^{4}\right)^{3 / 2}}+\mathcal{O}\left(\alpha^{2}\right)\right) .
$$

We can now extract the physics. First, for $\alpha=0$, there is a massless Goldstone pole corresponding to the spontaneously broken scale invariance. Moreover, there is a continuous spectrum of states corresponding to the branch cut $\left(4 l_{1}^{2} / l^{4},+\infty\right)$ on the complex Lorentzian $\tilde{p}_{L}^{2}=-\tilde{p}^{2}$ plane. Note that the threshold $M^{2}=4 l_{1}^{2} / l^{4}$ agrees precisely with that found in [5] by different means. Moreover, in the limit of vanishing VEV, $l_{1} \rightarrow 0$, we restore the two-point function imposed by conformal invariance for an operator of dimension 1 in three dimensions. Note in particular that in this limit the deformation parameter does not modify the two-point function, at least to the order we have computed it. This suggests that the $\mathcal{O}_{\Delta_{-}}$does not acquire an anomalous dimension when the marginal deformation is turned on, again at least to the order in $\alpha$ we have computed it and in the large- $N$ limit, for which the supergravity approximation holds.

\section{The fake superpotential as a quantum effective potential and multi- trace deformations}

We will now argue that, under certain circumstances, the fake superpotential that defines a given domain wall has a direct physical interpretation in the dual field theory as a 
quantum effective potential describing a marginal multi-trace deformation. As we will see, this interpretation requires that the bulk scalar fields admit two quantizations, as is the case for the $\mathrm{SL}(N, \mathbb{R}) / \mathrm{SO}(N)$ scalars that we have been discussing. In this case the onshell supergravity action plays two roles. More specifically, since $I\left[\phi_{-}\right]$and $\bar{I}\left[\bar{\phi}_{-}\right]$are the Legendre transform of each other and $\left\langle\mathcal{O}_{\Delta_{+}}\right\rangle=-\bar{\phi}_{-}$and $\left\langle\mathcal{O}_{\Delta_{-}}\right\rangle=\phi_{-}$, it follows that

i) $I\left[\phi_{-}\right]$is the generating functional of connected correlation functions of $\mathcal{O}_{\Delta_{+}}$and the quantum effective action for $\mathcal{O}_{\Delta_{-}}$.

ii) $\bar{I}\left[\bar{\phi}_{-}\right]$is the generating functional of connected correlation functions of $\mathcal{O}_{\Delta_{-}}$and the quantum effective action for $\mathcal{O}_{\Delta_{+}}$.

We have seen above that on a domain wall solution defined by the fake superpotential $W(\phi)$, the renormalized on-shell supergravity action computed with the standard Dirichlet boundary conditions is

$$
I=\int d^{d} x \sqrt{\gamma}\left(W(\phi)-W_{o}(\phi)\right)
$$

We will now show that this relation implies that the freedom in the fake superpotential, $W(\phi)$, is equivalent to computing the on-shell action with modified conformal boundary conditions and hence to a marginal multi-trace deformation of the boundary theory.

Multi-trace operators in any QFT that admits a large- $N$ limit and in the AdS/CFT correspondence are discussed in detail in appendix D. In the AdS/CFT correspondence, the effect of deforming the CFT action by a multi-trace operator $f\left(\mathcal{O}_{\Delta_{+}}\right)$, for the $\Delta_{+}$quantization, or by $\bar{f}\left(\mathcal{O}_{\Delta_{-}}\right)$, for the $\Delta_{-}$quantization, can be summarized in equations (D.12) and (D.18) respectively. It follows that the effect of a generic fake superpotential, $W(\phi)$, can be reproduced by computing the on-shell action with the superpotential $W_{o}(\phi)$ but with boundary conditions corresponding to a deformation

$$
\begin{aligned}
\bar{f}(\phi) & =W(\phi)-W_{o}(\phi), \quad \text { for } \quad \Delta_{-}, \\
f(\phi)-\phi f^{\prime}(\phi) & =W(\phi)-W_{o}(\phi), \quad \text { for } \quad \Delta_{+},
\end{aligned}
$$

or equivalently

$$
f(\phi)=-\phi \int \frac{d \phi}{\phi^{2}}\left(W(\phi)-W_{o}(\phi)\right), \quad \text { for } \quad \Delta_{+} .
$$

However, since the arguments of $f$ and $\bar{f}$ are the VEVs $\sigma$ and $\bar{\sigma}$ respectively, this interpretation of the fake superpotential is possible only when $W=W_{+}$for the $\Delta_{+}$quantization and $W=W_{-}$for the $\Delta_{-}$quantization. In summary, then

$$
\begin{aligned}
& \bar{f}(\phi)=W_{-}(\phi)-W_{o}(\phi)=\mathcal{O}\left(\phi^{3}\right), \quad \text { for } \quad \Delta_{-}, \\
& f(\phi)=-\phi \int \frac{d \phi}{\phi^{2}}\left(W_{+}(\phi)-W_{o}(\phi)\right)=-\frac{1}{2 l}\left(\Delta_{-}-\Delta_{+}\right) \phi^{I} \phi^{I}+\mathcal{O}\left(\phi^{3}\right), \quad \text { for } \quad \Delta_{+},
\end{aligned}
$$

where we have used the expansion

$$
W_{ \pm}(\phi)=-\frac{(d-1)}{\kappa^{2} l}-\frac{1}{2 l} \Delta_{ \pm} \phi^{I} \phi^{I}+\mathcal{O}\left(\phi^{3}\right)
$$




\begin{tabular}{|c|c|c|c|c|c|}
\hline$d$ & 2 & 3 & 4 & 5 & 6 \\
\hline$n$ & $n \geq 3$ & $3,4,5,6$ & 3,4 & 3 & 3 \\
\hline$\Delta_{-}$ & $2 / n$ & $1,3 / 4,3 / 5, \mathbf{1} / \mathbf{2}$ & $4 / 3, \mathbf{1}$ & $5 / 3$ & $\mathbf{2}$ \\
\hline
\end{tabular}

Table 3: The possible dimensions $d$ and conformal dimensions $\Delta_{-}$allowing for the interpretation of the fake superpotential as a multi-trace deformation of the dual theory. The dimensions in boldface saturate the unitarity bound.

But, if $W=W_{+}$, then $\phi^{2}$ corresponds to an irrelevant operator since $\Delta_{+}>d / 2$. It is therefore only for the $\Delta_{-}$quantization and for the fake superpotential $W_{-}$that an interpretation as a marginal multi-trace deformation of the boundary theory can arise. The above discussion can then be summarized in the following statement:

The marginal multi-trace deformations of a CFT admitting a holographic dual and corresponding to the $\Delta_{-}$quantization are in one-to-one correspondence with the possible fake superpotentials $W_{-}$of the dual bulk theory.

These fake superpotentials are determined by solving equation (1.8) as a differential equation for the fake superpotential. The conditions for such an interpretation of the fake superpotential impose strict restrictions on the dimension $d$ of the field theory as well as on the conformal dimension $\Delta_{-}$of the local operator. First, in order to get an $n$-trace marginal operator built from the single-trace operator $\mathcal{O}_{\Delta_{-}}$, we obviously need $\Delta_{-}=d / n$, where $n>2$ is an integer. The condition $n>2$ arises because the fake superpotential, $W_{-}$, cannot describe double-trace deformations since there is no freedom in its quadratic term. Moreover $\Delta_{-}$is bounded by $(d-2) / 2 \leq \Delta_{-}<d / 2$. The possible solutions of these conditions are summarized in table 3 . Note that only for $d=3$ is there an allowed $\Delta_{-}$ which is integer and yet it does not saturate the unitarity bound, namely $\Delta_{-}=1$.

\subsection{Triple-trace deformation of the Coulomb branch}

The field theory on the worldvolume of $N+1 M 2$-branes is an $\mathcal{N}=8$ (16 supercharges) superconformal field theory with $8(N+1)$ scalar and $8(N+1)$ fermionic degrees of freedom. Under the $\mathrm{SO}(8)$ R-symmetry group, the scalars, fermions and supersymmetries transform respectively as $\boldsymbol{8}_{v}, \boldsymbol{8}_{c}$ and $\boldsymbol{8}_{s}$ (see e.g. [51]). One of the $\mathcal{N}=8$ multiplets corresponds to the free theory describing the center of mass motion, while the remaining degrees of freedom parameterize the moduli space $\left(\mathbb{R}^{8}\right)^{N} / S_{N+1}$. This theory is believed to arise as the infra red fixed point of $\mathcal{N}=8$ supersymmetric Yang-Mills in three dimensions, while in the abelian case it can also be obtained by compactifying $\mathcal{N}=4$ supersymmetric Yang-Mills in four dimensions on a circle in the limit of vanishing circle radius. For the non-abelian case, however, this procedure is not well understood. In the large- $N$ limit this theory is holographically dual to eleven-dimensional supergravity on $A d S_{4} \times S^{7}$, whose massless sector is described by $\mathcal{N}=8$ gauged supergravity in four dimensions. The 70 scalars parameterizing the moduli space $E_{(7) 7} / \mathrm{SU}(8)$ of $\mathcal{N}=8$ gauged supergravity are holographically dual to BPS operators, which in the abelian case can be understood in 
terms the traceless bilinears of the 8 scalars and 8 fermions:

$$
\begin{aligned}
\mathcal{O}^{I J} & =\operatorname{Tr}\left(X^{I} X^{J}\right)-\frac{1}{8} \delta^{I J} \operatorname{Tr}\left(X^{K} X^{K}\right), \quad I, J, \ldots=1, \ldots, 8 \\
\mathcal{P}^{A B} & =\operatorname{Tr}\left(\lambda^{A} \lambda^{B}\right)-\frac{1}{8} \delta^{A B} \operatorname{Tr}\left(\lambda^{C} \lambda^{C}\right), \quad A, B, \ldots=1, \ldots, 8 .
\end{aligned}
$$

The 35 operators $\mathcal{O}^{I J}$ have conformal dimension $\Delta=1$ and transform in the $\mathbf{3 5}_{v}$ of $\mathrm{SO}(8)$, while the 35 scalars $\mathcal{P}^{A B}$ have conformal dimension $\Delta=2$ and transform in the $\mathbf{3 5}_{c}$. The 35 scalars parameterizing the $\mathrm{SL}(8, \mathbb{R}) / \mathrm{SO}(8)$ subspace of the scalar manifold are usually identified as dual to the dimension 1 operators $\mathcal{O}^{I J}$, in which case the supersymmetric domain wall solutions with non-trivial $\mathrm{SL}(8, \mathbb{R}) / \mathrm{SO}(8)$ scalars describe a uniform subsector of the Coulomb branch of the M2-brane theory [16, 14, 5]. Nevertheless, since the $\mathrm{SL}(8, \mathbb{R}) / \mathrm{SO}(8)$ scalars have a mass allowing two possible quantizations as we have discussed, and since in this case the $\Delta=1$ and $\Delta=2$ scalars both belong to the massless $\mathcal{N}=8$ supermultiplet and transform in $\mathrm{SO}(8)$ representations which are related by triality, it seems plausible that one could also identify these scalars as dual to the dimension 2 operators $\mathcal{P}^{I J} .^{22}$ In this case, as we have seen, the supersymmetric domain walls of the $\mathrm{SL}(8, \mathbb{R}) / \mathrm{SO}(8)$ sector correspond to deformations of the CFT Lagrangian.

When the $\operatorname{SL}(8, \mathbb{R}) / \mathrm{SO}(8)$ scalars are identified as dual to the dimension 1 operators $\mathcal{O}^{I J}$, the non-supersymmetric domain walls we have found describe the deformation of the uniform sector of the Coulomb branch, which corresponds to the supersymmetric superpotential, by a marginal triple-trace operator completely breaking supersymmetry. Such deformations have been discussed before in [52-55, 50. Indeed, as we have argued above, the fake superpotentials $W$ we have found compute the exact large- $N$ effective potential, given by

$$
V_{\text {eff. }}(\phi)=\bar{f}(\phi)=W(\phi)-W_{o}(\phi)=\left(C_{I J K}-C_{o I J K}\right) \phi^{I} \phi^{J} \phi^{K}+\mathcal{O}\left(\phi^{4}\right) .
$$

The higher order terms, which vanish when the cut-off is removed, correspond to irrelevant operators. Therefore, when the regulator is removed we are left with the triple-trace operator $\left(C_{I J K}-C_{O I J K}\right) \mathcal{O}^{I} \mathcal{O}^{J} \mathcal{O}^{K}$. This operator is classically marginal and remains marginal to leading order in $1 / N$ for any finite value of the dimensionless moduli $C_{I J K}$. However, the limit $C_{I J K} \rightarrow \infty$, corresponding to replacing $W(\phi ; \alpha)$ by $\widetilde{W}_{o}(\phi)$, does not commute with the cut-off removal, presumably due to the fact that we are working in the large- $N$ limit. Hence, we cannot simply drop the higher order irrelevant operators altogether, but instead we need the full fake superpotential. The role of these higher order terms can be understood by recalling that the these domain walls describe the Coulomb branch of the dual CFT and therefore, conformal invariance is spontaneously broken. This means that the coupling $C_{I J K}$ will run or, equivalently, the operator $\mathcal{O}^{3}$ will renormalize. Since there are no other free parameters in the fake superpotential other than the coupling $C_{I J K}$, the effect of the irrelevant operators can be interpreted as the running of the coupling $C_{I J K}$ or, equivalently, as the multiplicative renormalization of $\mathcal{O}^{3}$.

\footnotetext{
${ }^{22}$ This is special to four dimensions. In five or seven dimensions, one can unambiguously identify the $\mathrm{SL}(N, \mathbb{R}) / \mathrm{SO}(N)$ scalars from symmetries, by looking at the states of the relevant massless supermultiplet. I would like to thank Henning Samtleben for useful comments on this.
} 
Luckily we have already computed the exact fake superpotential for the one-scalar domain wall with $k=6$, which we will now consider as an example. For this case the full fake superpotential was given in (4.31). Using this we can now extract the exact large- $N$ anomalous dimensions $\gamma_{\mathcal{O}}$ and $\gamma_{\mathcal{O}^{3}}$. Starting from the kinetic term for the effective scalar field $\phi$ away from the origin of the moduli space [50 ( $\phi \neq 0$ since there is a non-zero VEV)

$$
K[\phi]=\frac{N^{2}}{8} \phi^{-1} \partial_{i} \phi \partial^{i} \phi
$$

and writing $\phi=\phi_{\Lambda}=\frac{\Lambda_{o}}{\Lambda} \tilde{\phi}\left(\frac{\Lambda_{o}}{\Lambda}\right)$, with $\tilde{\phi}\left(\frac{\Lambda_{o}}{\Lambda}\right)=\mathcal{O}\left(\Lambda^{0}\right)$ as $\Lambda \rightarrow \infty$, and identifying the UV cut-off with the AdS radial coordinate as $\Lambda=e^{r / l}$, we see that the multiplicative renormalization of $\mathcal{O}$ is given by

$$
Z_{\mathcal{O}}^{-1}(\Lambda)=\tilde{\phi}\left(\frac{\Lambda_{o}}{\Lambda}\right) / \tilde{\phi}(1) .
$$

It now follows from the first order equations (1.7) that

$$
\gamma_{\mathcal{O}}=\left.\Lambda \partial_{\Lambda} \log Z_{\mathcal{O}}(\Lambda)\right|_{\Lambda \rightarrow \infty}=-1-\left.l \frac{W^{\prime}}{\phi}\right|_{\phi \rightarrow 0} .
$$

Evaluating this using the fake superpotential (4.31) we obtain

$$
\gamma_{\mathcal{O}}= \begin{cases}0, & \alpha<\infty \\ 1, & \alpha \rightarrow \infty\end{cases}
$$

The dimension of the operator $\mathcal{O}$ therefore jumps from 1 for finite $\alpha$ to 2 in the $\alpha \rightarrow \infty$ limit. In this sense then the marginal triple-trace deformation interpolates between the two possible quantizations of the bulk scalar field, much like the situation described in e.g. 43, 48].

Similarly, we can now evaluate the anomalous dimension $\gamma_{\mathcal{O}^{3}}$. Since

$$
V_{\text {eff. }}=W-W_{o}=-\frac{2 \kappa}{l}\left(\frac{3}{2}\right)^{3 / 2} \frac{(\alpha+1)}{27} \phi^{3}+\mathcal{O}\left(\phi^{4}\right) \equiv-\frac{2 \kappa}{l}\left(\frac{3}{2}\right)^{3 / 2} \frac{(\alpha+1)}{27} Z_{\mathcal{O}^{3}}^{-1} \phi^{3},
$$

we find

$$
\gamma_{\mathcal{O}^{3}}=\left.\Lambda \partial_{\Lambda} \log Z_{\mathcal{O}^{3}}(\Lambda)\right|_{\Lambda \rightarrow \infty}= \begin{cases}0, & \alpha<\infty, \\ 1, & \alpha \rightarrow \infty .\end{cases}
$$

Note that the running coupling is simply given by $\bar{\alpha}+1=Z_{\mathcal{O}^{3}}^{-1}(\alpha+1)$ and so $\beta_{\alpha}=(\alpha+1) \gamma_{\mathcal{O}^{3}}$. It follows that, in agreement with the expectation in [53], the triple-trace operator $\mathcal{O}^{3}$ remains marginal for all finite values of $\alpha$ and in the large- $N$ limit. In the $\alpha \rightarrow \infty$ limit, however, $\mathcal{O}^{3}$ has dimension 4 and not the naively expected dimension 6 . It nevertheless remains an irrelevant operator in this limit.

\section{Acknowledgments}

I am grateful to Professor Jerome Gauntlett for a helpful conversation on the significance of curvature singularities in M-theory backgrounds. I would also like to thank Dimitry Belyaev for useful comments. 


\section{A. Explicit form of the domain wall metric for $W(\phi ; \alpha)$, to first order in $\alpha-\alpha_{o}$ and for general $k$}

In this appendix we give the explicit form of the domain wall backgrounds corresponding to the fake superpotential (4.13) to first order in $\alpha-\alpha_{o}$. In order to discuss all values of $k$ at once, it is convenient to trade the radial coordinate $r$ in (1.1) for the single scalar field $X$. Another advantage of this radial coordinate is that it is directly related to the scalar field $\phi$ via (4.2) and so we only need to determine the domain wall metric. This can be done by solving the flow equations (1.7), which now become

$$
\begin{aligned}
\dot{X} & =\kappa^{2}\left(\frac{8-k}{2 k}\right) X^{2} \partial_{X} W, \\
\partial_{X} A & =-\left(\frac{k}{8-k}\right) \frac{W}{X^{2} \partial_{X} W} .
\end{aligned}
$$

Inserting the fake superpotential (4.13) and integrating these equations to first order in $\left(\alpha-\alpha_{o}\right)$ we find

$$
\begin{gathered}
\frac{d r}{l}=-\frac{8}{(8-k)} \frac{X^{\frac{k}{(8-k)}-1} d X}{\left(X^{\frac{8}{(8-k)}}-1\right)}\left(1-\frac{3\left(\alpha-\alpha_{o}\right)}{2 k(8-k)} X^{\frac{k}{(8-k)}-3}\left(X^{\frac{8}{(8-k)}}-1\right)\left(k X^{\frac{8}{(8-k)}}+8-k\right)\right. \\
\left.+\mathcal{O}\left(\left(\alpha-\alpha_{o}\right)^{2}\right)\right) \\
e^{A}=\frac{8}{(8-k)} \frac{X}{\left(X^{\frac{8}{(8-k)}}-1\right)}\left\{1+\frac{\left(\alpha-\alpha_{o}\right)}{2 k(8-k)}\left[k X^{\frac{4(k-2)}{(8-k)}}+\frac{2 k(8-k)}{(k-4)}\left(X^{\frac{4(k-4)}{(8-k)}}-1\right)\right.\right. \\
\left.\left.-(8-k) X^{\frac{4(k-6)}{(8-k)}}-2(k-4)\right]+\mathcal{O}\left(\left(\alpha-\alpha_{o}\right)^{2}\right)\right\} .
\end{gathered}
$$

Note that for $\alpha=\alpha_{o}$ one recovers the supersymmetric solutions of [14, 5].

\section{B. Uplifting the MTZ black hole to eleven dimensions}

The gravity-scalar system (1.2) in four dimensions with a single scalar field and the potential (4.24) was also considered in [19], where a topological black hole with non-trivial scalar hair was found. It was also pointed out in [19] that this scalar potential acquires a very simple form in the conformal frame defined by

$$
\tilde{\psi} / 3=\tanh (\psi / 3), \quad \tilde{g}_{\mu \nu}=\cosh ^{2}(\psi / 3) g_{\mu \nu},
$$

where $\tilde{\psi}=\sqrt{\frac{3 \kappa^{2}}{2}} \tilde{\phi}$. In this frame the action takes the form

$$
S=\int_{\mathcal{M}} d^{4} x \sqrt{-\tilde{g}}\left(\frac{1}{2 \kappa^{2}} \tilde{R}-\frac{1}{2} \tilde{g}^{\mu \nu} \partial_{\mu} \tilde{\phi} \partial_{\nu} \tilde{\phi}-\frac{1}{12} \tilde{R} \tilde{\phi}^{2}-\tilde{V}(\tilde{\phi})\right),
$$

where

$$
\tilde{V}(\tilde{\phi})=-\frac{3}{\kappa^{2} l^{2}}\left(1-\left(\kappa^{2} / 6\right)^{2} \tilde{\phi}^{4}\right)
$$


The scalar field is now conformally coupled to gravity and the $\tilde{\phi}^{4}$ potential ensures that the scalar field equations are conformally invariant. Quite remarkably this system admits an exact instanton solution [56].

The four-dimensional black hole found in [19], which we will refer to as the MTZ black hole, $\operatorname{reads}^{23}$

$$
\begin{aligned}
d s_{4}^{2} & =\frac{r(r+2 G \mu)}{(r+G \mu)^{2}}\left\{-\left(\frac{r^{2}}{l^{2}}-\left(1+\frac{G \mu}{r}\right)^{2}\right) d t^{2}+\left(\frac{r^{2}}{l^{2}}-\left(1+\frac{G \mu}{r}\right)^{2}\right)^{-1} d r^{2}+r^{2} d \sigma^{2}\right\}, \\
\phi & =\sqrt{\frac{3}{4 \pi G}} \tanh ^{-1}\left(\frac{G \mu}{G \mu+r}\right),
\end{aligned}
$$

where $d \sigma^{2}$ is the metric on a two-dimensional compact manifold, $\Sigma_{2}$, of constant negative curvature. This means that $\Sigma_{2} \cong \mathbb{H}_{2} / \Gamma$, where $\mathbb{H}_{2}$ is the hyperbolic plane and $\Gamma$ is a freely acting discrete subgroup of the isometry group $O(2,1)$. This black hole has curvature singularities at $r=0$ and $r=-2 G \mu$. The range of the radial coordinate is taken $r>0$ for $\mu>0$ and $r>-2 G \mu$ for $\mu<0$. In either case the curvature singularity is hidden behind a horizon located at $r_{+}=\frac{l}{2}(1+\sqrt{1+4 G \mu / l})$, provided

$$
\mu>-\frac{1}{4 G}
$$

Note also that $\phi \geq 0$ for $\mu>0$ and $\phi \leq 0$ for $\mu<0$. The mass of the black hole is given by

$$
M=\frac{\sigma}{4 \pi} \mu,
$$

where $\sigma$ is the area of $\Sigma_{2}$, and its Hawking temperature is

$$
T_{H}=\frac{1}{2 \pi l}\left(\frac{2 r_{+}}{l}-1\right)
$$

As in section 5, we can uplift this black hole to eleven dimensions using the reduced ansatz (5.5). In terms of the scalar field

$$
X=e^{\psi / 3}=e^{\kappa \phi / \sqrt{3}}=\left(\frac{2 G \mu+r}{r}\right)^{1 / 8},
$$

and the quantity

$$
\tilde{\Delta}=X \cos ^{2} \theta+X^{-3} \sin ^{2} \theta
$$

the eleven-dimensional metric is

$$
d \hat{s}_{11}^{2}=\tilde{\Delta}^{2 / 3} d s_{4}^{2}+4 l^{2} \tilde{\Delta}^{-1 / 3}\left\{X^{3}\left[\left(\cos ^{2} \theta+X^{-4} \sin ^{2} \theta\right) d \theta^{2}+\sin ^{2} \theta d \phi_{4}^{2}\right]+X^{-1} \cos ^{2} \theta d \Omega_{5}^{2}\right\}
$$

\footnotetext{
${ }^{23}$ We have kept the notation of [19] here, hoping this will cause no confusion. Note, in particular, that the radial coordinate, $r$, here is not related to the radial coordinate in the domain wall metric (1.1), and, as usual, $\kappa^{2}=8 \pi G$.
} 
and the four-form field strength reads

$$
\begin{aligned}
\hat{F}_{(4)}= & \left\{\frac{r^{4}(r+2 G \mu)^{2}}{l(r+G \mu)^{4}}\left[2 X^{2} \cos ^{2} \theta+X^{-2}\left(1+2 \sin ^{2} \theta\right)\right] d r\right. \\
& \left.+2 G \mu l X\left[1-\frac{r^{2}}{l^{2}}\left(1+\frac{G \mu}{r}\right)^{-2}\right] \cos \theta \sin \theta d \theta\right\} \wedge d t \wedge \bar{\epsilon}_{(2)},
\end{aligned}
$$

where $\bar{\epsilon}_{(2)}$ is the volume form on $\Sigma_{2}$.

\section{Computation of the holographic two-point functions}

In this appendix we give the details of the computation of the holographic two-point functions for the domain walls defined by the fake superpotential (4.13), which have been constructed explicitly in appendix A.

We start with the supersymmetric solutions corresponding to $\alpha=\alpha_{o}$. For this case the first two equations in (7.8) read respectively

$$
\begin{gathered}
\left(X^{2} \partial_{X}^{2}+\frac{(16-5 k) X^{\frac{8}{(8-k)}}+(5 k-32)}{(8-k)\left(X^{\frac{8}{(8-k)}}-1\right)} X \partial_{X}-q^{2} X^{\frac{4(k-4)}{(8-k)}}\right) e_{j}^{i}=0 \\
\left(X^{2} \partial_{X}^{2}+\frac{k(16-5 k) X^{\frac{8}{(8-k)}}+(8-k)(5 k-32)}{(8-k)\left(k X^{\frac{8}{(8-k)}}+8-k\right)} X \partial_{X}-q^{2} X^{\frac{4(k-4)}{(8-k)}}\right) \omega=0
\end{gathered}
$$

where $q^{2}=p^{2} l^{2}(8-k)^{2} / 64$. We have managed to solve these equations analytically only for the case $k=4$, and so we will focus on this case. Having solved the supersymmetric case, we can then obtain the solution for the non-supersymmetric fake superpotential $W(\phi ; \alpha)$ perturbatively in $\alpha-\alpha_{o}$. Since for $k=4$ we have $\alpha_{o}=0$, the expansion is actually in $\alpha$. For $k=4$ then, to first order in $\alpha$, the first two equations in (7.8) take the form

$$
\begin{aligned}
& \left\{\left(X^{2} \partial_{X}^{2}-\frac{\left(X+3 X^{-1}\right)}{\left(X-X^{-1}\right)} X \partial_{X}-q^{2}\right)\right. \\
& \left.+\frac{3 \alpha}{4}\left(\left(X^{2}-X^{-2}\right)\left(X^{2} \partial_{X}^{2}+X \partial_{X}\right)+\frac{q^{2}}{3}\left(X^{2}-X^{-2}+8 \ln X\right)\right)+\mathcal{O}\left(\alpha^{2}\right)\right\} e_{j}^{i}=0 \\
& \left\{\left(X^{2} \partial_{X}^{2}-\frac{\left(X-3 X^{-1}\right)}{\left(X+X^{-1}\right)} X \partial_{X}-q^{2}\right)\right. \\
& +\frac{3 \alpha}{4}\left(\left(X^{2}-X^{-2}\right) X^{2} \partial_{X}^{2}+\left[X^{2}-X^{-2}+\frac{4}{3}\left(\frac{\left(X+X^{-1}\right)^{2}+2}{X+X^{-1}}\right)^{2}\right] X \partial_{X}\right. \\
& \left.\left.+\frac{q^{2}}{3}\left(X^{2}-X^{-2}+8 \ln X\right)\right)+\mathcal{O}\left(\alpha^{2}\right)\right\} \omega=0
\end{aligned}
$$


The solutions of these equations, which are non-singular as $X \rightarrow \infty$, are respectively

$$
\begin{aligned}
e_{j}^{i}= & e_{(0) j}{ }_{j} X^{-a}\left[(1+a) X+(1-a) X^{-1}\right] \\
& \left\{1+\frac{\alpha}{8 a^{2}}\left(a^{2}\left[(2 a-1) X^{2}+(2 a+1) X^{-2}\right]-\frac{8\left(a^{2}+1\right)}{(a+1) X\left[(1+a) X+(1-a) X^{-1}\right]}\right.\right. \\
& \left.-4 \ln X\left[\frac{\left(2 a^{2}-2 a+1\right)(1+a) X+\left(2 a^{2}+2 a+1\right)(1-a) X^{-1}}{(1+a) X+(1-a) X^{-1}}-\left(a^{2}-1\right) a \ln X\right]\right) \\
& \left.+\mathcal{O}\left(\alpha^{2}\right)\right\}, \\
\omega= & \omega_{(0)} X^{-a}\left[(1+a) X-(1-a) X^{-1}\right] \\
& \left\{1+\frac{\alpha}{8 a^{2}}\left(a^{2}\left[(2 a-3) X^{2}+(2 a+3) X^{-2}\right]-\frac{8\left[\left(a^{2}+1\right)-\left(a^{2}-1\right)\left(a^{2}-2\right)\right]}{(a+1) X\left[(1+a) X-(1-a) X^{-1}\right]}\right.\right. \\
& \left.-4 \ln X\left[\frac{\left(2 a^{2}-2 a+1\right)(1+a) X-\left(2 a^{2}+2 a+1\right)(1-a) X^{-1}}{(1+a) X-(1-a) X^{-1}}-\left(a^{2}-1\right) a \ln X\right]\right) \\
& \left.+\mathcal{O}\left(\alpha^{2}\right)\right\},
\end{aligned}
$$

where $a=\sqrt{1+q^{2}}$ and $e_{(0)}{ }_{j}^{i}$ and $\omega_{(0)}$ are arbitrary functions of $q$.

It is now straightforward to evaluate $E$ and $\Omega$ using these exact solutions. To isolate the desired coefficients $E_{(3)}$ and $\Omega_{(1)}$ we first need to determine the terms $E_{(1)}, E_{(2)}$ as well as $\Omega_{(0)}$ and subtract them from the exact solutions for $E$ and $\Omega$. This can be done using the first order equations (7.11) and the dilatation operator as described in section 7 . One easily finds

$$
\begin{aligned}
& E_{(1)}=\quad \Omega_{(0)}=0, \\
& E_{(2)}=\frac{4}{l}\left(a^{2}-1\right) e^{-2 A}=\frac{1}{4 l}\left(a^{2}-1\right)\left(X-X^{-1}\right)^{2}\left[1-\frac{\alpha}{4}\left(X^{2}-X^{-2}+8 \ln X\right)+\mathcal{O}\left(\alpha^{2}\right)\right\} .
\end{aligned}
$$

Subtracting these from the exact solutions for $E$ and $\Omega$ we finally determine

$$
\begin{aligned}
& E_{(3)}=-\frac{1}{l}\left(\frac{\kappa}{\sqrt{2}}\right)^{3} \phi_{B}^{3} a\left(a^{2}-1\right)\left(1+\alpha \frac{\left(3 a^{2}-1\right)}{2 a^{3}\left(a^{2}-1\right)}+\mathcal{O}\left(\alpha^{2}\right)\right), \\
& \Omega_{(1)}=\frac{1}{l} \frac{\kappa}{\sqrt{2}} \phi_{B} a^{-1}\left(a^{2}-1\right)\left(1+\alpha \frac{\left(3 a^{2}-1\right)\left(2 a^{2}-1\right)}{2 a^{3}\left(a^{2}-1\right)}+\mathcal{O}\left(\alpha^{2}\right)\right),
\end{aligned}
$$

which allow one to evaluate the exact one-point functions with linear sources (7.17) and hence the two-point functions.

\section{Multi-trace deformations in the large- $N$ limit and the AdS/CFT cor- respondence}

Multi-trace operators in the AdS/CFT correspondence have been studied extensively [4350]. Before we discuss such operators in the context of the AdS/CFT correspondence, however, it is useful to review some generic field theoretic properties of multi-trace operators in quantum field theories that admit a large- $N$ limit. These properties are independent of 
the AdS/CFT correspondence and will allow us to incorporate multi-trace operators in the AdS/CFT correspondence in a very elegant way.

We will first for completeness repeat the field theory argument given in [50], applicable to any quantum field theory that admits a large- $N$ limit. Let $\mathcal{O}(x)$ be a local gauge-invariant single-trace operator, with the trace taken in the adjoint for concreteness, normalized such that $\langle\mathcal{O}\rangle=\mathcal{O}\left(N^{0}\right)$ as $N \rightarrow \infty$. The generating functional of connected correlators, $W[J]$ is $\mathcal{O}\left(N^{2}\right)$ and so it is convenient to write $W[J]=N^{2} w[J]$. In terms of the field theory action, $S[\phi]$, then

$$
e^{-N^{2} w[J]}=\int[d \phi] e^{-S[\phi]-N^{2} \int d^{d} x J(x) \mathcal{O}(x)} .
$$

Now define

$$
\sigma(x) \equiv\langle\mathcal{O}\rangle_{J}=\frac{\delta w[J]}{\delta J} .
$$

The effective action $\Gamma[\sigma]=N^{2} \bar{\Gamma}[\sigma]$ is given by

$$
e^{-N^{2} \bar{\Gamma}[\sigma]}=\int[d J] e^{-N^{2} w[J]+N^{2} \int d^{d} x J(x) \sigma(x)},
$$

and

$$
J=-\frac{\delta \bar{\Gamma}[\sigma]}{\delta \sigma}
$$

Consider now the deformed action $S_{f}[\phi]=S[\phi]+N^{2} \int d^{d} x f(\mathcal{O})$. Then,

$$
\begin{aligned}
e^{-N^{2} w_{f}\left[J_{f}\right]} & =\int[d \phi] e^{-S[\phi]-N^{2} \int d^{d} x\left(J_{f} \mathcal{O}+f(\mathcal{O})\right)} \\
& =\int[d \phi] e^{-S[\phi]-N^{2} \int d^{d} x\left(J \mathcal{O}+f(\mathcal{O})-f^{\prime}(\sigma) \mathcal{O}\right)} \\
& \stackrel{\approx}{ } \rightarrow e^{-N^{2} w[J]} e^{-N^{2} \int d^{d} x\left(f(\sigma)-\sigma f^{\prime}(\sigma)\right)}
\end{aligned}
$$

where we introduced

$$
J \equiv J_{f}+f^{\prime}(\sigma)
$$

in the second line in order to remove the linear term from $f(\mathcal{O})$ so that large- $N$ factorization can be used in the last step. It follows that in the large- $N$ limit, the generating functional of connected correlators in the deformed theory is given by

$$
\begin{aligned}
w_{f}\left[J_{f}\right] & =\bar{\Gamma}_{f}[\sigma]+\int d^{d} x J_{f} \sigma \\
& =w[J]+\left.\int d^{d} x\left(f(\sigma)-\sigma f^{\prime}(\sigma)\right)\right|_{\sigma=\delta w[J] / \delta J} .
\end{aligned}
$$

Moreover,

$$
\begin{aligned}
e^{-N^{2} \bar{\Gamma}_{f}[\sigma]} & =\int\left[d J_{f}\right] e^{-N^{2} w_{f}\left[J_{f}\right]+N^{2} \int d^{d} x J_{f} \sigma} \\
& =\int[d J] e^{-N^{2} w_{f}[J]} e^{-N^{2} \int d^{d} x\left(f(\sigma)-\sigma f^{\prime}(\sigma)\right)} e^{N^{2} \int d^{d} x\left(J-f^{\prime}(\sigma)\right) \sigma} \\
& =e^{-N^{2} \bar{\Gamma}[\sigma]-N^{2} \int d^{d} x f(\sigma)},
\end{aligned}
$$


where we have used $\left[d J_{f}\right]=[d J]$. Therefore,

$$
\bar{\Gamma}_{f}[\sigma]=\bar{\Gamma}[\sigma]+\int d^{d} x f(\sigma),
$$

or equivalently

$$
V_{\text {eff }}^{f}(\sigma)=V_{\text {eff }}(\sigma)+N^{2} f(\sigma) .
$$

These results rely only on the existence of a large- $N$ limit and are independent of the AdS/CFT correspondence. However, they allow for an elegant reformulation of Witten's [43] prescription for incorporating multi-trace operators in the AdS/CFT correspondence. Recall, that in the supergravity approximation, one computes the renormalized on-shell supergravity action $I[\phi]$, which is a functional of $\phi_{-}$only since $\phi_{+}$is expressed in terms of $\phi_{-}$by requiring regularity in the interior. Equipped with the renormalized on-shell supergravity action, the AdS/CFT prescription for multi-trace operators can be stated as follows, depending on the $\Delta_{ \pm}$quantization:

For the $\Delta=\Delta_{+}$quantization, one identifies the generating functional of the undeformed theory as

$$
\left.W[J] \equiv I\left[\phi_{-}\right]\right|_{\phi_{-}=J} .
$$

For the deformed theory, Witten's prescription amounts to setting

$$
\begin{aligned}
W_{f}\left[J_{f}\right] & \left.\equiv I\left[\phi_{-}\right]\right|_{\phi_{-}=\phi_{-}^{*}\left(J_{f}\right)} \\
& =\left.I\left[\phi_{-}\right]\right|_{\phi_{-}=J}+\left.\int d^{d} x\left(f(\sigma)-\sigma f^{\prime}(\sigma)\right)\right|_{\sigma=\delta I\left[\phi_{-}\right] /\left.\delta \phi_{-}\right|_{\phi_{-}=J}},
\end{aligned}
$$

where $\phi_{-}^{*}\left(J_{f}\right)$ is the solution to

$$
\phi_{-}=J_{f}+f^{\prime}\left(\frac{\delta I\left[\phi_{-}\right]}{\delta \phi_{-}}\right) .
$$

Noting that $\phi_{-}=J$ and $\frac{\delta I\left[\phi_{-}\right]}{\delta \phi_{-}}=\sigma$, this equation is precisely equation (D.6), thus justifying this prescription for the incorporation of multi-trace operators in the AdS/CFT correspondence.

For the $\Delta=\Delta_{-}$quantization, one merely needs to replace $I\left[\phi_{-}\right]$with the Legendre transform $\bar{I}\left[\bar{\phi}_{-}\right]$defined in (6.21), (6.22), (6.23). Namely, the generating functional of the undeformed theory is now given by

$$
\left.\bar{W}[\bar{J}] \equiv \bar{I}\left[\bar{\phi}_{-}\right]\right|_{\bar{\phi}_{-}=\bar{J}},
$$

while the effective action is given by the on-shell action

$$
\bar{\Gamma}[\bar{\sigma}]=I[\bar{\sigma}] .
$$

For the deformed theory then

$$
\left.\bar{W}_{\bar{f}}\left[\bar{J}_{\bar{f}}\right] \equiv \bar{I}\left[\bar{\phi}_{-}\right]\right|_{\bar{\phi}_{-}=\bar{\phi}_{-}^{*}\left(\bar{J}_{\bar{f}}\right)},
$$


where $\bar{\phi}_{-}^{*}\left(\bar{J}_{\bar{f}}\right)$ is the solution to

$$
\bar{\phi}_{-}=\bar{J}_{\bar{f}}+\bar{f}^{\prime}\left(\frac{\delta \bar{I}\left[\bar{\phi}_{-}\right]}{\delta \bar{\phi}_{-}}\right) .
$$

The effective action is then given by

$$
\begin{aligned}
\bar{\Gamma}_{f}[\bar{\sigma}] & =\bar{W}\left[\bar{J}_{\bar{f}}\right]-N^{2} \int d^{d} x \bar{J}_{\bar{f}} \bar{\sigma} \\
& =I[\bar{\sigma}]+N^{2} \int d^{d} x \bar{f}(\bar{\sigma}) .
\end{aligned}
$$

\section{References}

[1] M. Cvetič and H.H. Soleng, Supergravity domain walls, Phys. Rept. 282 (1997) 159 hep-th/9604090.

[2] D.Z. Freedman, C. Núñez, M. Schnabl and K. Skenderis, Fake supergravity and domain wall stability, Phys. Rev. D 69 (2004) 104027 hep-th/0312055.

[3] A. Celi, A. Ceresole, G. Dall'Agata, A. Van Proeyen and M. Zagermann, On the fakeness of fake supergravity, Phys. Rev. D 71 (2005) 045009 hep-th/0410126.

[4] K. Skenderis and P. K. Townsend, Gravitational stability and renormalization-group flow, Phys. Lett. B 468 (1999) 46 hep-th/9909070.

[5] I. Bakas, A. Brandhuber and K. Sfetsos, Domain walls of gauged supergravity, $m$-branes and algebraic curves, Adv. Theor. Math. Phys. 3 (1999) 1657 hep-th/9912132.

[6] M. Zagermann, $N=4$ fake supergravity, Phys. Rev. D 71 (2005) 125007 hep-th/0412081.

[7] K. Skenderis and P.K. Townsend, Hidden supersymmetry of domain walls and cosmologies, Phys. Rev. Lett. 96 (2006) 191301 [hep-th/0602260].

[8] K. Skenderis, private communication.

[9] P.K. Townsend, Positive energy and the scalar potential in higher dimensional (super)gravity theories, Phys. Lett. B 148 (1984) 55.

[10] O. DeWolfe, D.Z. Freedman, S.S. Gubser and A. Karch, Modeling the fifth dimension with scalars and gravity, Phys. Rev. D 62 (2000) 046008 hep-th/9909134.

[11] S.S. Gubser, Curvature singularities: the good, the bad and the naked, Adv. Theor. Math. Phys. 4 (2002) 679 hep-th/0002160.

[12] I. Papadimitriou and K. Skenderis, AdS/CFT correspondence and geometry, hep-th/0404176.

[13] V.L. Campos, G. Ferretti, H. Larsson, D. Martelli and B.E.W. Nilsson, A study of holographic renormalization group flows in $D=6$ and $D=3$, JHEP 06 (2000) 023 hep-th/0003151.

[14] M. Cvetič, S.S. Gubser, H. Lu and C.N. Pope, Symmetric potentials of gauged supergravities in diverse dimensions and coulomb branch of gauge theories, Phys. Rev. D 62 (2000) 086003 hep-th/9909121.

[15] M. Cvetič, H. Lu, C.N. Pope and A. Sadrzadeh, Consistency of Kaluza-Klein sphere reductions of symmetric potentials, Phys. Rev. D 62 (2000) 046005 hep-th/0002056. 
[16] P. Kraus, F. Larsen and S. P. Trivedi, The Coulomb branch of gauge theory from rotating branes, JHEP 9903 (1999) 003 hep-th/9811120.

[17] D.Z. Freedman, S.S. Gubser, K. Pilch and N.P. Warner, Continuous distributions of D3-branes and gauged supergravity, JHEP 07 (2000) 038 hep-th/9906194.

[18] I. Bakas and K. Sfetsos, States and curves of five-dimensional gauged supergravity, Nucl. Phys. B $573(2000) 768$.

[19] C. Martinez, R. Troncoso and J. Zanelli, Exact black hole solution with a minimally coupled scalar field, Phys. Rev. D 70 (2004) 084035 hep-th/0406111.

[20] B. de Wit and H. Nicolai, The consistency of the $S^{7}$ truncation in $D=11$ supergravity, Nucl. Phys. B 281 (1987) 211.

[21] H. Nastase, D. Vaman and P. van Nieuwenhuizen, Consistent nonlinear K K reduction of $11 D$ supergravity on $A d S_{7} \times S^{4}$ and self-duality in odd dimensions, Phys. Lett. B 469 (1999) 96 hep-th/9905075.

[22] H. Nastase, D. Vaman and P. van Nieuwenhuizen, Consistency of the $A d S_{7} \times S^{4}$ reduction and the origin of self-duality in odd dimensions, Nucl. Phys. B 581 (2000) 179 hep-th/9911238.

[23] P. Breitenlohner and D.Z. Freedman, Stability in gauged extended supergravity, Ann. Phys. $(N Y) 144(1982) 249$

[24] I.R. Klebanov and E. Witten, AdS/CFT correspondence and symmetry breaking, Nucl. Phys. B 556 (1999) 89 hep-th/9905104.

[25] Dr. E. Kamke, Differentialgleichungen Lösungsmethoden und Lösungen, Chelsea Publishing Company (1971).

[26] E. S. Cheb-Terrab and A. D. Roche, Abel ODEs: equivalence and integrable classes, Comput. Phys. Commun. 130 (2000) 204 math-ph/0001037;

E.S. Cheb-Terrab and A.D. Roche, An Abel ordinary differential equation class generalizing known integrable classes, European J. Appl. Math. 142003217, math.GM/0002059;

V.M. Boyko, Symmetry, equivalence and integrable classes of Abel's equations, Proceedings of the Institute of Mathematics of the NAS of Ukraine 50, part 1, (2004) 47 nlin-sys/0404020.

[27] M.J. Duff and J.T. Liu, Anti-de Sitter black holes in gauged $N=8$ supergravity, Nucl. Phys. B 554 (1999) 237 hep-th/9901149.

[28] M. Cvetič et al., Embedding AdS black holes in ten and eleven dimensions, Nucl. Phys. B $\mathbf{5 5 8}(1999) 96$ hep-th/9903214.

[29] J. de Boer, E.P. Verlinde and H.L. Verlinde, On the holographic renormalization group, JHEP 08 (2000) 003 hep-th/9912012.

[30] M. Bianchi, D.Z. Freedman and K. Skenderis, How to go with an RG flow, JHEP 08 (2001) 041 hep-th/0105276.

[31] I. Papadimitriou and K. Skenderis, Correlation functions in holographic RG flows, JHEP 10 (2004) 075 hep-th/0407071.

[32] M. Henningson and K. Skenderis, The holographic Weyl anomaly, JHEP 07 (1998) 023 hep-th/9806087. 
[33] V. Balasubramanian and P. Kraus, A stress tensor for anti-de Sitter gravity, Commun. Math. Phys. 208 (1999) 413 hep-th/9902121.

[34] P. Kraus, F. Larsen and R. Siebelink, The gravitational action in asymptotically AdS and flat spacetimes, Nucl. Phys. B 563 (1999) 259 hep-th/9906127.

[35] S. de Haro, S.N. Solodukhin and K. Skenderis, Holographic reconstruction of spacetime and renormalization in the AdS/CFT correspondence, Commun. Math. Phys. 217 (2001) 595 hep-th/0002230.

[36] M. Bianchi, D.Z. Freedman and K. Skenderis, Holographic renormalization, Nucl. Phys. B 631 (2002) 159 hep-th/0112119.

[37] D. Martelli and W. Muck, Holographic renormalization and Ward identities with the Hamilton-Jacobi method, Nucl. Phys. B 654 (2003) 248 hep-th/0205061.

[38] K. Skenderis, Lecture notes on holographic renormalization, Class. and Quant. Grav. 19 (2002) 5849 hep-th/0209067.

[39] D.Z. Freedman, S.D. Mathur, A. Matusis and L. Rastelli, Correlation functions in the $C F T(d) / A d S(d+1)$ correspondence, Nucl. Phys. B $546(1999) 96$ hep-th/9804058.

[40] O. DeWolfe and D.Z. Freedman, Notes on fluctuations and correlation functions in holographic renormalization group flows, hep-th/0002226.

[41] W. Muck, Correlation functions in holographic renormalization group flows, Nucl. Phys. B 620 (2002) 477 hep-th/0105270.

[42] M. Bianchi, M. Prisco and W. Muck, New results on holographic three-point functions, JHEP 11 (2003) 052 hep-th/0310129.

[43] E. Witten, Multi-trace operators, boundary conditions and AdS/CFT correspondence, hep-th/0112258.

[44] M. Berkooz, A. Sever and A. Shomer, Double-trace deformations, boundary conditions and spacetime singularities, JHEP 05 (2002) 034 hep-th/0112264].

[45] W. Muck, An improved correspondence formula for AdS/CFT with multi- trace operators, Phys. Lett. B 531 (2002) 301 hep-th/0201100;

[46] P. Minces, Multi-trace operators and the generalized AdS/CFT prescription, Phys. Rev. D 68 (2003) 024027 hep-th/0201172.

[47] A. Sever and A. Shomer, A note on multi-trace deformations and AdS/CFT, JHEP 07 (2002) 027 hep-th/0203168.

[48] S.S. Gubser and I.R. Klebanov, A universal result on central charges in the presence of double-trace deformations, Nucl. Phys. B 656 (2003) 23 hep-th/0212138.

[49] O. Aharony, M. Berkooz and B. Katz, Non-local effects of multi-trace deformations in the AdS/CFT correspondence, JHEP 10 (2005) 097 hep-th/0504177.

[50] S. Elitzur, A. Giveon, M. Porrati and E. Rabinovici, Multitrace deformations of vector and adjoint theories and their holographic duals, JHEP 02 (2006) 006 hep-th/0511061.

[51] R. Corrado, K. Pilch and N.P. Warner, An $N=2$ supersymmetric membrane flow, Nucl. Phys. B 629 (2002) 74 hep-th/0107220. 
[52] T. Hertog and K. Maeda, Black holes with scalar hair and asymptotics in $N=8$ supergravity, JHEP 07 (2004) 051 hep-th/0404261.

[53] T. Hertog and G.T. Horowitz, Towards a big crunch dual, JHEP 07 (2004) 073 hep-th/0406134.

[54] T. Hertog and G.T. Horowitz, Designer gravity and field theory effective potentials, Phys. Rev. Lett. 94 (2005) 221301 hep-th/0412169.

[55] T. Hertog and G.T. Horowitz, Holographic description of AdS cosmologies, JHEP 04 (2005) 005 hep-th/0503071.

[56] S. de Haro, I. Papadimitriou and A. Petkou, Conformally coupled scalars, instantons and vacuum instability in $A d S(4)$ hep-th/0611315. 